\title{
Solar Energy Technical Training \\ Directory
}

\section{MASTER}

George Corcoleotes

Katherine Kramer

Kevin O'Connor

Solar Energy Research Institute

June 1979

Prepared for the

United States Department of Energy

Contract No. EG-71-C-01-4042

A product of the
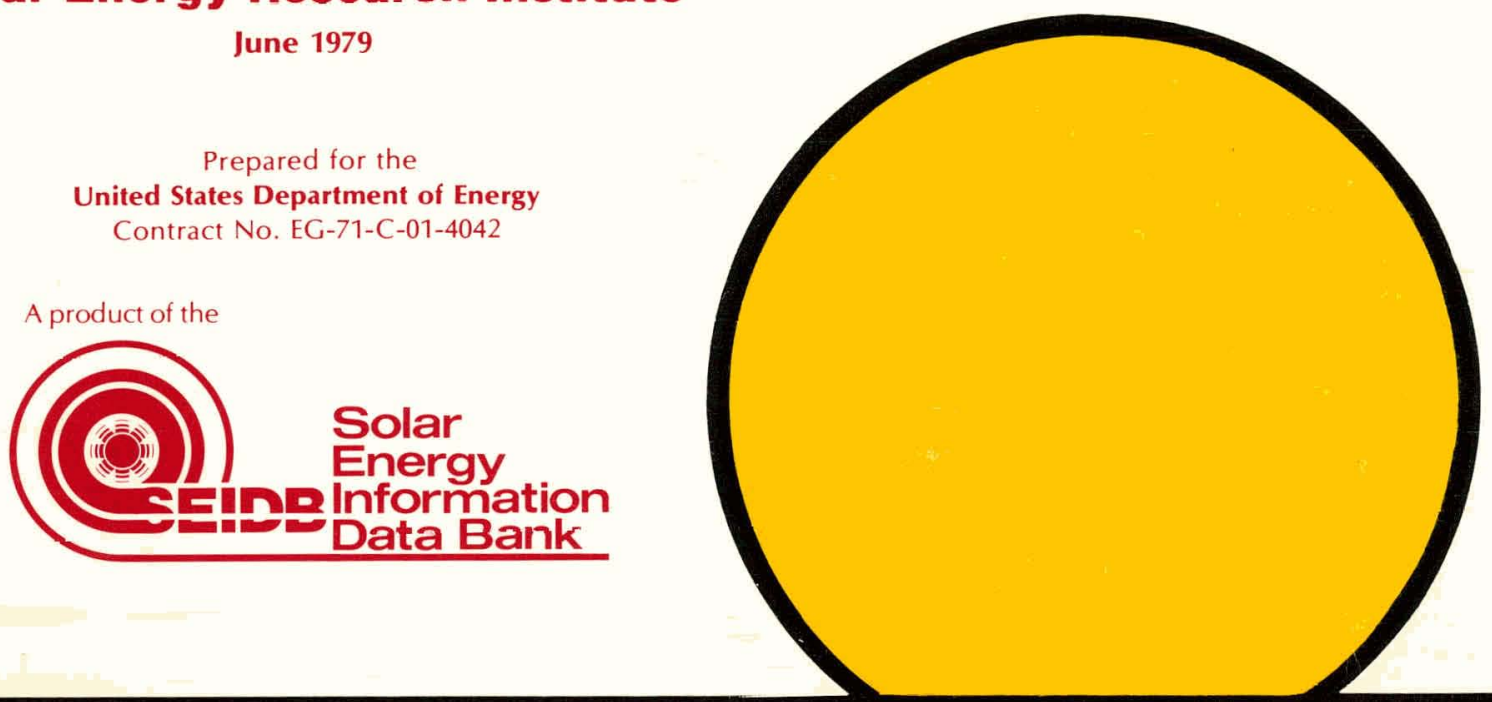


\section{DISCLAIMER}

This report was prepared as an account of work sponsored by an agency of the United States Government. Neither the United States Government nor any agency Thereof, nor any of their employees, makes any warranty, express or implied, or assumes any legal liability or responsibility for the accuracy, completeness, or usefulness of any information, apparatus, product, or process disclosed, or represents that its use would not infringe privately owned rights. Reference herein to any specific commercial product, process, or service by trade name, trademark, manufacturer, or otherwise does not necessarily constitute or imply its endorsement, recommendation, or favoring by the United States Government or any agency thereof. The views and opinions of authors expressed herein do not necessarily state or reflect those of the United States Government or any agency thereof. 


\section{DISCLAIMER}

Portions of this document may be illegible in electronic image products. Images are produced from the best available original document. 
For additional copies contact:

National Solar Heating and Cooling Information Center

P.O. Box 1607

Rockville, Maryland 20850

Call toll free (800) 523-2929

In Pennsylvania (800) 462-4983

In Alaska \& Hawaii (800) 523-4700

Microfiche Copies Available From:

National Technical Information Service

U.S. Department of Commerce

5285 Port Royal Road

Springfield, VA 22161

(\$3.00 each)

\section{NOTICE}

This report was prepared as an account of work sponsored by the United States Government. Neither the United States nor the United States Department of Energy, nor any of their employees, nor any of their contractors, subcontractors, or their employees, make any warranty, express or implied, or assumes any legal liability or responsibility for the accuracy, completeness or usefulness of any information, apparatus, production or process disclosed, or represents that its use would not infringe privately owned rights.

Printed in the United States of America. 


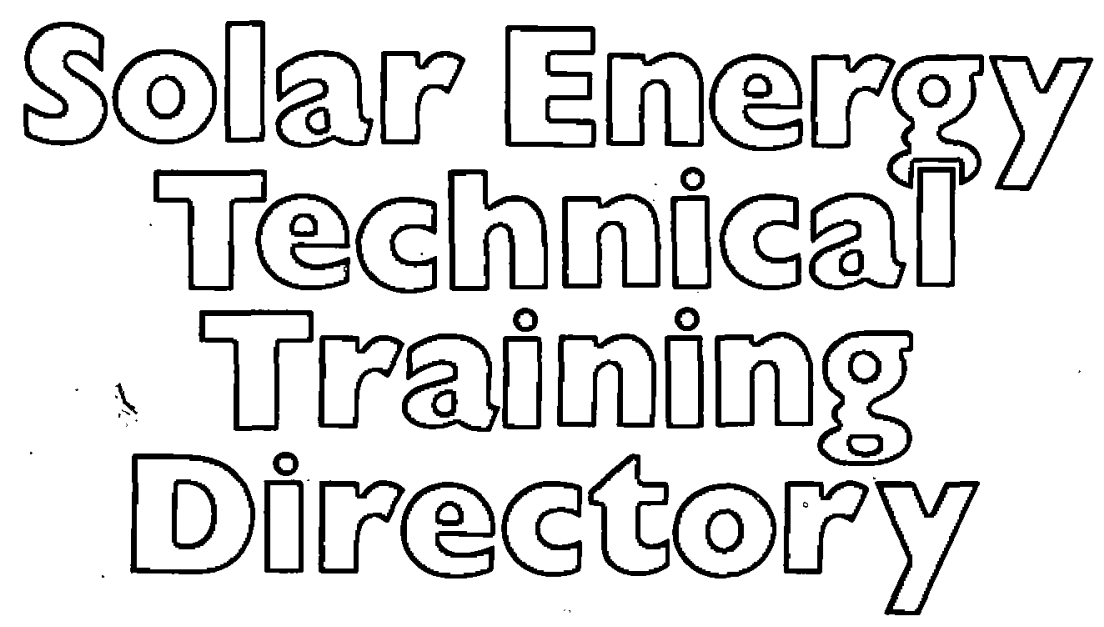

George Corcoleotes
Katherine Kramer
Kevin O'Connor

Solar Energy Research Institute

June 1979

Prepared for the

United States Department of Energy

Contract No. EG-71-C-01-4042
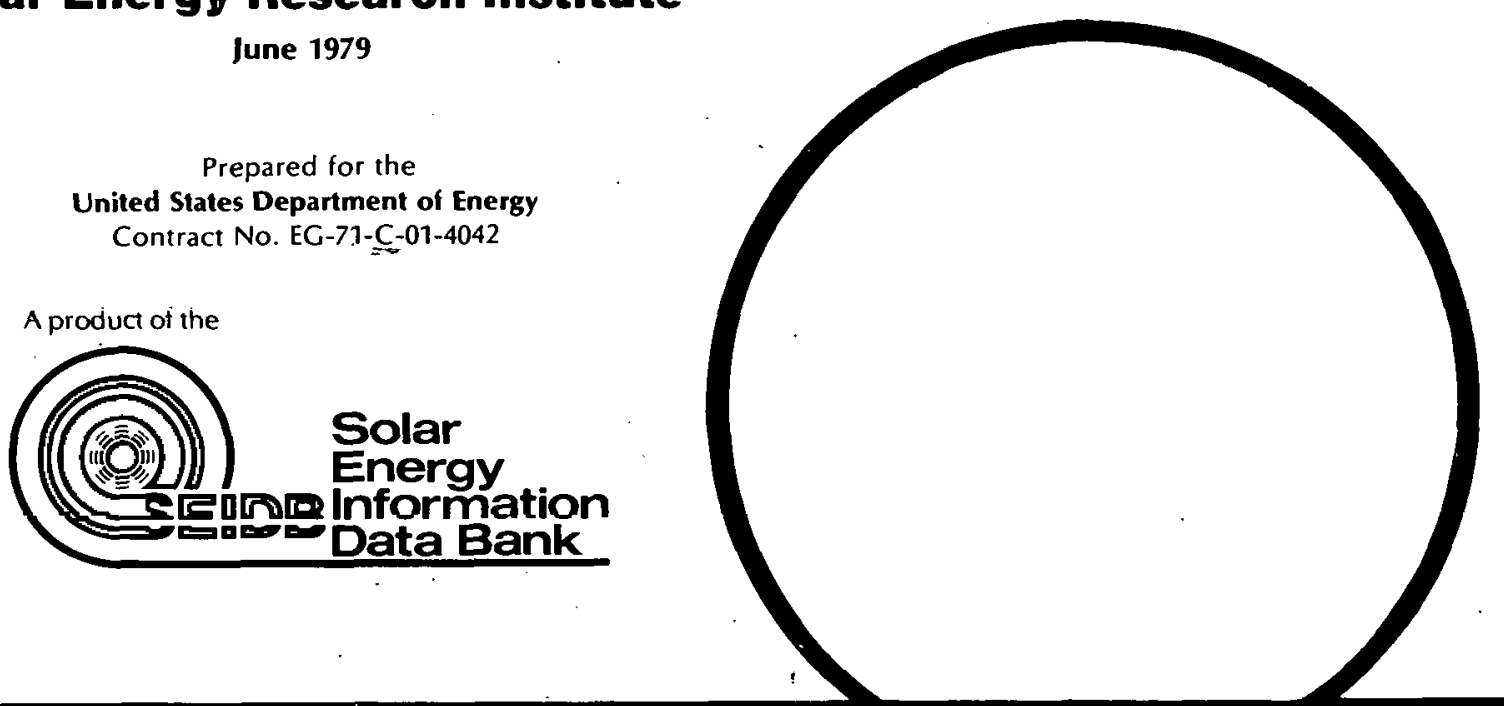
For additional copies contact:

National Solar Heating and Cooling Information Center

P.O. Box 1607

Rockville, Maryland 20850

Call toll free (800) 523-2929

In Pennsylvania (800) 462-4983

In Alaska \& Hawaii (800) 523-4700

Mir.rntiōthe Conpiēss $\Delta$ vailāhle Frn̄m: National Technical Information Service

U.S. Department of Commerce 5285 Port Royal Road Springfield, VA 22161

(\$3.00 each)

NOTICE

This report was prepared as an account of work sponsored by the United States Government. Neither the United States nor the United States Department of Energy, nor any of their employees, nor any of their contractors, subcontractors, or their employees, make any warranty, express or implied, or assumes any legal liability or responsibility for the accuracy, completeness or usefulness of any information, apparatus, production or process disclosed, or represents that its use would not infringe privately owned rights.

Printed in the United States of America. 


\section{Preface}

This Directory was prepared as part of Task \#4228, Vocational Training Programs, of the Academic Programs Branch of the Solar Energy Research Institute (SERI). The report reflects the current status of available solar energy educational offerings in the technical training area. The completeness of the Directory reflects the most thorough effort to gather national information on solar educational courses, programs and curricula.

Approved for:

Solar Energy Research Institute

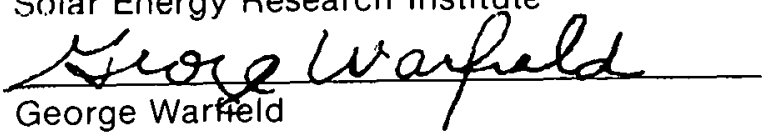

Director for

Technology Dissemination

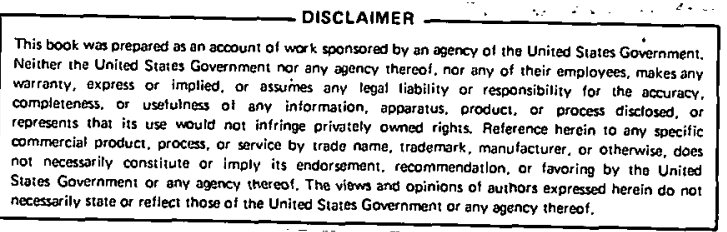




\title{
Solar Energy Technical Training Directory
}

\author{
First Edition
}

\section{Introduction}

- The Solar Energy Technical Training Directory is prepared by the Solar Energy Research Institute (SERI) as a subset of the first edition of the National Solar Energy Education Directory. This technical training directory is intended to be a quick reference for students, counselors, researchers, and others having an interest in solar technical training programs.

Most institutions currently reporting technical training programs are vocational-technical schools and community or junior colleges. Information was gathered from a 1978 national survey of postsecondary institutions. Only those institutions which indicated offering solar technical training activities are included in the Solar Energy Technical Training Directory.

All survey information is maintained in the computerized Education Data Base produced by SERI's Academic and International Programs and Information Systems Divisions in cooperation with the Office of U.S. Congressman George E. Brown, Jr., and the Congressional Solar Coalition. The Education Data Base is one of many data bases being developed for the Solar Energy Information Data Bank, mandated by Congress to provide solar information to Government, the scientific and educational communities, and the private sector. SERI is leading the development of the Solar Energy Information Data Bank on behalf of the U.S. Department of Energy.

\section{Note to Users}

Secondary sources of information used in this report are indicated by an asterisk $\left({ }^{*}\right)$ in the directory text. The list of institutions to which the surveys were addressed was obtained from the National Center for Education Statistics. Revisions or additions to be included in future editions of this publication may be addressed to Academic Programs Branch, Attn: George Corcoleotes, Solar Energy Research Institute, 1536 Cole Blvd., Golden CO 80401. Telephone (303) 231-1831 Numbers in parentheses to the right of institution names are for identification and should be used when submitting revisions or additions.

\section{Directory Organization}

The Directory lists institutions alphabetically by state. Each listing includes an institution address and telephone number, solar programs or curricula offered and detailed solar course information. An alphabetical index of institutions appears at the back of the Directory.

\footnotetext{
*The National Solar Energy Education Directory may be ordered from the Superintendent of Documents, U.S. Government Printing Office, Washington, D.C., 20402; stock number 061-00000210-3, price $\$ 4.75$. TheNational Directory includes complete information (indexed, 279 pages) on solar courses, programs, and curricula offered by all post-secondary iristilutions.
} 


\section{ALABAMA, ALASKA \\ None}

\section{ARIZONA \\ Yavapai College [1079] \\ Prescott, Arizona 86301 \\ (602) 445-7300 \\ Programs and Curricula \\ Solar Energy Technology \\ Contact: \\ Minkler, L./ Beverly, G./ Strom, L. (602) $445-7300$ \\ Students Taking or Completing Otfering: \\ Do-it-yourself Homeowner, \\ Installer-Residential (Solar Systems)}

Solar Related Courses

Here Comes the Sun

Instructor:

Minkler, Lyle

(602) 445-5261

Course Number: PAS100

Department: Science

Program or Curriculum: Solar Energy Technology

Credits:

Duration: 5

Contact Hours:

5 Weeks, $3.0 \mathrm{hrs}$ per week

Classroom:

Topics Covered Extensively: Heat and Energy Transfer: Intro. to Solar Energy; Solar Economics; Solar Collector Evaluation/Design

Number of Times Taught:

Average Enrollment: 28

Methane, Wind-Electricity, Wood-Alternate Energy

Instructor:

Beverly, Gary

(602) 445-7300

Course Number:

PHS109

Department:

Science.

Program or Curriculum: Solar Energy Technology

Credits: 1

Duration:

Contact Hours:

5 Weeks, $3.0 \mathrm{hrs}$ per week 15

Topics Covered Extensively: Alternate Energy Sources;

Appropriate Technology; Biomass Conversion; Elec'l

Generation, Small Scale

Number of Times Taught: 4

Average Enrollment: 14

Solar Cookers

Instructor:

Course Number:

Minkler, Lyle

(602) 445-5264

Department:

PHS105

Srience

Program or Curriculum: Solar Energy Technology

Credits:

1

Duration:

Contact Hours:

Weeks, $3.0 \mathrm{hrs}$ per week

Design

Solar Greenhouse

Instructor:

Beverly, Gary

(602) 445-7300

Course Number: PHS107

Department: Science

Program or Curriculum: Solar Energy Technology

Credits: 1

Duration:

Contact Hours:

Topics Covered Extensively: Appropriate Technology;

Energy Storage; Heat and Energy Transfer; Passive Solar Technology; Solar System Components; Solar Systems

Design; Space Heating

Number of Times Taught: 5

Average Enrollment: $\quad 16$
Solar Heating, Air and Water Systems

Instructor:

Minkler, Lyle

Course Number:

(602) 445-5264

Program or Curriculum: Solar Energy Technology

Credits:

1

Duration

Contact Hours:

5 Weeks, 3.0 hrs per week 15

Topics Covered Extensively: Appropriate Technology;

Energy Storage; Solar System Components; Solar Systems Design

Number of Times Taught: 1

Average Enrollment: $\quad 15$

Solar Heating, Passive and Hybrid Systems

Instructor: Frerking, Mike

Course Number: PHS102

Department: Science

Program or Curriculum: Solar Energy Technology

Credits: 1

Duration: $\quad 5$ Weeks, $3.0 \mathrm{hrs}$ per week

Contact Hours: 15

Topics Covered Extensively: Energy Storage; Heat and Energy Transfer; Passive Solar Technology; Solar System Components; Solar Home Construction; Solar Collector

Evaluation/Designi; Space Heating

Number of Times Taught: 1

Average Enrollment: $\quad 9$

Solar Heating, Retrofit Systems

Instructor: Minkler, Lyle

(602) 445-7300

Course Number: PHS104

Department: Science

Program or Curriculum: Solar Energy Technology

Credits: 1

Duration: $\quad 5$ Weeks, $3.0 \mathrm{nrs}$ per week

Contact Hours: $\quad 15$

Topics Covered Extensively: Appropriate Technology; Energy Storage; Heat and Energy Transfer; Solar System Components; Solar Economics; Solar Law/Legislation; Solar Collector Evaluation/Design; Solar Systems Design; Space Heating

Solar Hot Water

Instructor:

Beverly, Gary

(602) 445-7300

Course Number: PHS103

Department: . Science

Program or Curriculum: Solar Energy Technology

Credits:

1

Contact Hours:

5 Weeks, 3.0 hrs per week

extensivel

Energy Storage; Heat and Energy Transfer; Plumbing

Techniques; Solar System Components; Solar Economics;

Solar Collector Evaluation/Design; Solar Systems Design;

Domestic Hot Water

Number of Times Taught: 1

Average Enrollment:

4

Solar Laboratory 121

Instructor:

Minkler, Lyle

(602) $445-7300$

Course Number: $\quad$ PHS121

Department: Science

Program or Curriculum: Solar Energy Technology

Credits:

Duration:

Contact Hours:

Classroom:

Laboratory:

Solar Energy Technology

6 Weeks, 4.5 hrs per week

27

6

Topics Covered Extensively: Solar Systems Installation;

Solar Systems Maintenance; Solar Systems Testing and

Evaluation; Domestic Hot Water; Space Heating; Space

Cooling

Numbor of Times Taught: 1

Average Enrollment: 25 
Solar Laboratory 122

Instructor:

Minkler, Lyle

(602) $44 b-1300$

$\begin{array}{ll}\text { Course Number: } & \text { PHS122 } \\ \text { Department: : } & \text { Science }\end{array}$

Program or Curriculum: Solar Energy Technology.

Credits:

Duration:

6 Weeks, $4.5 \mathrm{hrs}$ per week

Contact Hours:

Classroom:

27

6

Laboratory:

21

Topics Covered Extensively: Solar Systems Installation;

Solar Systems Maintenance; Solar Systems Testing and

Evaluation, Dómestic Hot Water; Space Heating; Space

Cooling

Number of Times Taught: 1

Average Enrollment: 25

Solar Laboratory 123

Minkler, Lyle

(602) 445-7300

PHS123

Course Number:

Science

Department:

Solar Energy Technology

Program or Curriculum:

Credits:

1

Duration:

6 Weeks, $4.5 \mathrm{hrs}$ per week

Contact Hours:

27

6

Classivurl:

21

Topics Covered Extensively: Solar Systems Installation;

Solar Systems Maintenance; Solar Systems Testing and

Evaluation; Domestic Hot Water; Space Heating; Space

Cooling

Number of Times Taught: 1

Average Enrolliment: $\quad 2 b$

Solar Laboratory 124

Instructor:

Minkler, Lyle

(602) $445-7300$

PHS124

Course No. Science

Department:

Solar Energy Technology

Credits:

Duration:

Contact Hours:

Classroom: 6

Laboratory: 21

Topics Covered 'Extensively: Solar Systems Installation:

Solar Systems Maintenance; Solar Systems Testing and

Evaluation; Domestic Hot Water; Space Heating; Space

Cooling

Number of Times Taught: 1

Average Enrollment: 25

Solar Laboratory 125

Instructor:

Minkler, Lyle

(602) $445-7300$

$\begin{array}{ll}\text { Course Number: } & \text { PHS125 } \\ \text { Department: } & \text { Science }\end{array}$

Program or Cuirriculum: Solar Energy Technology

Credits: 1

Duration:

1 .

Contact Hours:

6 Weeks, 4.5 hrs per week

Classroom:

27

Laboratory: 21

Topics Covered Extensively: Solar Systems Installation;

Solar Systems Maintenance; Solar.Systems Testing and

Evaluation; Domestic Hot Water; Space Heating; Space

Cooling

Number of Times Taught: 1

Average Enrollment: 25

\section{ARKANSAS \\ Mississippi County Community College [12860] Blytheville, Arkansas 72315 \\ (501) 762-1020}

Programs and Curricula

Solar Energy Technology

Degree:

Contact:

AD, Applied Science in Solar

Technology

Hughes, G. Edward/ Benson, Chris (501) $762-1020$

Students Taking or Completing Offering:

Solar Technician

Solar Related Courses

Solar Technology Cooperative Education

Instructor: Benson, C.M.

(501) $762-1020$

Course Number: $\quad \mathbf{5 8 9 7 0}$

Department: Applied Science

Program or Curriculum: Solar Energy Technology

Credits:

Student Level: $\quad$ Freshman or Sophomore

Duration: $\quad 15$ Weeks, 6.0 hrs per week

Contact Hours: $\quad 90$

Tuplcs Covered Extensively: Hlumbıng lechnıques; Solar Systems Installation; Solar Syslems Mainterlance; Solar Systems Testing and Evaluation; Domestic Hot Water; Elec'l Generation, Central; Elec'l Generation, Small Scale; Process Heat, Agricultural; Process Heat, Industrial; Space Heating; Space Cooling

Number of Times Taught: 1

Average Enroliment: 10

Solar Technology 1

Instructor:

Benson, C.M

(501) $762-1020$

Course Number: $\quad 58003$

Department: Applied Science

Program or Currlculum: Solar Energy I echnology

Credits:

Student Level:

Duration:

Contact Hours:

Classroom:

Freshman or Sophomore

15 Weeks, $3.0 \mathrm{hrs}$ per week

45

Topics Covered Extensively: Appropriate Technology;

Energy Storage; Heat and Energy Transfer; Intro. to Solar

Energy; Passive Solar Technology; Solar Collector

Evaluation/Design; Domestic Hot Water; Space Heating;

Gpace Couling

Number of Times Taught: 1

Average Enroliment 20

Solar Technology I Lab.

Instructor:

Benson, C.M.

Course Number:

(501) $762-1020$

58001

Department: Applied Science

Program or Curriculum: Solar Energy Technology

Credits:

Student Level: $\quad$ Freshman or Sophomore

Duration: $\quad 15$ Weeks, $2.0 \mathrm{hrs}$ per week

Contact Hours: $\quad 30$

Laboratory: $\quad 30$

Topics Covered Extensively; Energy Storage; Materials

Research; Plumbing Techniques; Solar System

Components; Solar Systems Testing and Evaluation;

Domestic Hot Water; Space Heating; Space Cooling

Number of Times Taught: 1

Average Enrollment:

Solar Technology II

Instructor:

Course Number:

Department:

10

or Curriculum: Solar Energy Technology

Credits:

Student Level: $\quad$ Freshman or Sophomore
Benson, C.M.

(501) $762-1020$

58203 
Duration:

Contact Hours:

15 Weeks, $3.0 \mathrm{hrs}$ per week

Classroom:

Topics Covered Extensively: Energy Storage; Heat and Energy Transfer; Photovoltaics; Solar System Components; Solar Economics; Solar Collector Evaluation/Design: Elec'l Generation, Central; Elec'l Generation, Small Scale; Space Heating: Space Cooling

Number of Times Taught: 1

Average Enrollment: 20

Solar Technology II Lab. Instructor:

Benson, C.M (501) $762-1020$ 58201

Course Number: Applied Science

Department: Solar Energy Technology Credits:

Student Level:

Duration:

Contact Hours:

Freshman or Sophomore 15 Weeks, $2.0 \mathrm{hrs}$ per week 30

Laboratory:

Topics Covered Extensively: Materials Research; Photovoltaics; Plumbing Techniques; Solar System Components: Solar Systems Design; Solar Systems Testing and Evaluation; Elec'l Generation, Central; Elec'l Generation, Small Scale; Space Cooling

Number of Times Taught: 1

Average Enrollment: $\quad 10$

Solar Technology III

Instructor:

Course Number:

Benson, C.M.

(501) 762-1020

Department:

58403

Applied Science

Program or Curriculum: Solar Energy Technology

Credits:

3

Student Level:

Freshman or Sophomore

Duration:

Contact Hours:

15 Weeks, $3.0 \mathrm{hrs}$ per week

45

45

Classroom:

ively: Energy Storage; Photovoltaics;

Plumbing Techniques; Solar Energy Policy Development;

Solar Economics; Solar Systems Design; Domestic Hot

Water; Elec'l Generation, Central; Elec'l Generation, Small

Scale; Process Heat, Agricultural; Process Heat, Industrial;

Space Heating; Space Cooling

Number of Times Taught: 1

Average Enrollment: 20

Solar Technology III Laboratory

Instructor: Benson, C.M.

(501) $762-1020$

$\quad 58401$

Department: Applied Science

Program or Curriculum: Solar Energy Technology

Crodits:

Student. Level:

1

Duration:

Contact Hours:

Freshman or Sophomore

Laboratory:

15 Weeks, $2.0 \mathrm{hrs}$ per week

30

30

Topics Covered Extensively: Energy Storage; Material

Materials Rescarch; Photovoltaics; Plumbing Techniques;

Solar System Components; Solar Systems Design; Solar

Systems Installation; Solar Systems Maintenance; Solar

Systems Testing and Evaluation; Domestic Hot Water;

Elec'l Generation, Central; Elec'l Generation, Small Scale;

Process Heat, Agricultural; Process Heat, Industrial; Space

Heating; Space Cooling:

Number of Times Taught: 1

Average Enrollment: 10

Solar Topics

Instructor:

Benson, C.M

(501) $762-1020$

Course Number

58700

Department: Applied Science

Prngram or Eurriculum: Solar Energy Techinuluyy

Credits:

Freshman or Sophomore
Duration:

Contact Hours:

15 Weeks, $2.0 \mathrm{hrs}$ per week

Classroom:

Laboratory:

30

15

Topics Covered Extensively: Energy Storage; Photovoltaics:

Plumbing Techniques; Solar Energy Policy Development;

Solar System Components; Solar Economics; Solar Home

Construction; Solar Law/Legislation; Solar Collector

Evaluation/Design; Solar Systems Design; Solar Systems

Installation; Solar Systems Maintenance; Solar Systems

Testing and Evaluation

Number of Times Taught: 1

Average Enrollment: 5

\section{CALIFORNIA}

Antioch University West [90520]

650 Pine Street

San Francisco, California 94108

Programs and Curricula

*Solar Energy and Design

Degree:

BA, MS, Environmental Studies and

Contact: Appropriate Technology Nelson, Lynn

Solar Related Courses

(415) $956-1688$

*Courses in Design; Construction of Solar Systems

Instructor:

Department:

Olkowski, Helga

Program or Curriculum: "Solar Energy and Design

Topics Covered Extensively: Passive Solar Technology Solar System Cormponents; Solar Home Construction; Solar Collector Evaluation/Design; Solar Systems Design; Space Heating; Space Cooling

\section{Cabrillo College [1124]}

Aptos, California 95003

(408) 425-6000

Programs and Curricula

Solar Technology

Degree:

AD, Science

Contact:

Burton, Dave

(408) 425-6304

Students Taking or Completing Offering

Solar Technician

Solar Related Courses

Alternate Energy Systems (Solar Technology)

Course Number: CET60ABCD

Dcpartment: Indust. - Elect. Tech

Program or Curriculum: Solar Technology

Credits

Student Level: $\quad$ All Levels

Duration: $\quad 16$ Weeks, $15.0 \mathrm{hrs}$ per week

Contact Hours: $\quad 240$

Classroom: $\quad 80$

Laboratory: $\quad 160$

Topics Covered Extensively: Alternate Energy Sources:

Materials Research; Solar System Components; Solar

Home Construction; Solar Collector Evaluation/Design;

Solar Systems Design; Solar Systems Installation

Applications of Solar Energy in Agriculture

Course Number: CET61

Program or Curriculum: Solar Technology

Credits: 3

Student Level: $\quad$ All Levels

Duration: $\quad 16$ Weeks, $5.0 \mathrm{hrs}$ per week

Contact Hours: $\quad 80$

Classroom: $\quad 32$

Laboratory:

Toplcs Covered Extensively: Biomass Conversion; Energy

Conservation; Energy Conversion; Energy Storage; Heat

and Energy Transfer; Passive Solar Technology; Solar 
System Components; Solar Home Construction; Solar Collector Evaluation/Design; Solar Systems Design; Solar Systems Installation; Process Heat, Agricultural

\section{Solar Architecture}

Course Number: CET62

Program or Curriculum: Solar Technulogy

Credits: 3

Stuident I evel All Levels

Duration 16 Weeks, 5.0 hrs per week

Contac̈t Hours: 80

Classroom: 32

Laboratory 48

Topics Covered Extensively: Energy Conservation; Passive Solar-Technology; Solar System Components; Solar. Economics; Solar Collector Evaluation/Design; Solar Systems Design; Space Heating

Solar Electronics

Course Number:

\section{CET53}

Department: Indus. - Elect. Tech.

Program or Curriculum: Solar Technology

Credits:

Student Level:

All Levels

16 Weeks, $3.0 \mathrm{hrs}$ per week

Contact Hours: 48

Topics Covered Extensively: Photovoltalcs; Solar System

Components; Domestic Hot Water; Elec'l Generation,

Central; Elec'l Generation. Small Scale

Solar Energy In Agricullure

Course Number: ' CET54

Program or Curriculum: Solar Technology

Credits: 3

Student Level:

All Levels

Duration:

Contact Hours:

16 Weeks, 4.0 hrs per week

Classroom:

64

32

Laboratory:

32

Topics Covered Extensively: Biomass Conversion; Intro.

to Solar Energy; Solar Home Construction; Solar Collector Evaluation/Design; Solar Systems Design; Process Heat,

Agricultural; Space Heating

Solar Energy in Building Design

Course Number: CET52

Deparlinêt: Industrial - Elcctrical Technology

Program or Curriculum: Solar Technology

Credits:

3

Student Level:

Duration:

Contact Hours:

All Levels

16 Weeks, 4.0 hrs.per week 64

Topics Covered Extensively: Energy Conservation; Energy

Conversion; Energy Storage; Heat and Energy Transfer;

Passive Solar Technology; Plumbing Techniques; Solar

System Components; Solar Economics; Solar Collector

Evaluation/Design; Solar Systems Design

Solar Energy Technology and Farication

Course Numbcr: CET60ABCD

Department: Industrial - Electrical Technology

Program or Curriculum: Solar Technolngy

Credits:

Stuḍent laevel:

Duration:

Contact 1\%ours:

Laboratory:

8

All Levels

16 Weeks, $12.0 \mathrm{hrs}$ per week

192

192

Topics Covered Extensively: Solar System Components;

Solar Systems Design; Solar Systems Installation; Solar

Systems Maintenance; Solar Systems Testing and Evalua-

tion; Domestic Hot Water; Swimming Pool Heating; Space

Heating

\section{Solar Retrofitting and Weatherizing}

Course Number:

CET51

Department:

Industrial - Electrica! Technology

Program or Curriculum: Solar Technology

Credits:

2

Student Level:

Duration:

All Levels

Contact Hours

16 Weeks, $3.0 \mathrm{hrs}$ per week 48

Topics Covered Extensively: Energy Conversion; Energy
Storage: Solar Collector Evalualioni/Design; Solar Systems

Desıgn; Solar Systems Installation

\section{California Stafe University-Sonoma [1156] \\ Rohnert Park, California 94928 \\ (707) 664-2880}

Programs and Curricula

Solar Energy Technical Training Program

Degree: Certificate

Contact: Roy Irving

Students Taking or Completing Offering:

Solar Technician

Solar Related Courses

Independent Studies in Solar Energy

Instructor: Roy Irving

(707) 664-2577

Program or Curriculum: Solar Energy Technician

Credits:

Student Level: Junior or Senior

Duration: 15 weeks, 12 hrs per week

Contact Hours: $\quad 180$

Independent Study: $\quad 180$

Number of Times Taught: 2

Average Enrollment: 20

Solar Energy, Direct Uses

Instructor:

Roy Irving

(707) 664-2577

Course Number ENSP 337

Program or Curriculum: Solar Energy Technical

Credits Training Program

Duration:

Contact Hours:

Classivom:

Junior or Senior

Topics Covered Extensively. Alternate Energy Sources:

Energy Conservation; Energy Storage; Heat and Energy

Transfer; Intro. to Solar Energy; Passive Solar Technology;

Solar System Components; Domestic Hot Water; Space Heating

Number of Times Taught: 14

Average Enrollment: 35

Advanced Solar Energy Seminar

Instructor:

Roy Irving

Course Number

(707) $664-2577$

Solar Energy Tec.hnic.al

L'rèdits

Training Program

Student Level:

3

Duration:

Contact Hours:

Junior or Senior

Classroom: 45

Topics Covered Extensively: Heat and EnergyTransfer; Passive

Solar Technology; Solar Economics; Solar Law/Legislation

Solar System Design; Domestic Hot Water; Space Heating

Number of Times Taught: 1

Average Enrollment: 28

Center for Employment Training

[90350]

425 So. Market St.

San Jose, California 95113

Solar Related Courses

*Building Malntenance

Iristructor:

Rodriguez, Rudolph

Duration:

6 Weeks 
Topics Covered Extensively: Solar System Components; Solar Collector Evaluation/Design; Solar Systems

Design; Solar Systems Installation

\section{Cerro Coso Community College [10111] Ridgecrest, California 93555 (714) 375-5001 \\ Programs and Curricula \\ "Solar Engineering Technology \\ Degree: \\ AD, Applied Science - Solar \\ Contact: Engineering Technology Dodge, Dick (714) $375-5001$ \\ Students Taking or Completing Offering: Solar Technician}

\section{Chaffey College [1163] \\ Alta Loma, California 91701 (714) $987-1737$}

Solar Related Courses

* Solar Energy 1

Instructor:

Course Number:

Rothwell, Robert

Department:

Duration :

Contact Hours:

507

Indus. Tech.

12 Weeks, $6.0 \mathrm{hrs}$ per week

Topics Covered Extensively: Intro. to Solar Energy:

Passive Solar Technology; Solar System Components;

Solar Collector Evaluation/Design; Solar Systems Design;

Domestic Hot Water; Swimming Pool Heating; Space

Heating; Space Cooling

\section{*Solar Energy II}

$\begin{array}{ll}\text { Instructor: } & \text { Rothwell, Robert } \\ \text { Course Number: } & 508 \\ \text { Department: } & \text { Indus. Tech. } \\ \text { Duration: } & 12 \text { Weeks, } 6.0 \text { hrs per week } \\ \text { Topics Covered Extensively: Energy Conversion; Energy } \\ \text { Storage; Heat and Energy Transfer; Photovoltaics; Solar } \\ \text { System Components; Solar Collector Evaluation/Design; } \\ \text { Solar Systems Design; Solar Systems Installation }\end{array}$

\section{Coastline Community College [29027]}

Fountain Valley, California 92708

(714) 963-0811

Programs and Curricula

* Solar Technician

Students Taking or Completing Offering:

Solar Technician

Solar Related Courses

* Solar Seminar

"Ten Courses in Energy Management

Program or Curriculum: Solar Technician

\section{Cosumnes River College \\ Sacramento, California \\ (916) 421-1000}

Programs and Curricula

Environmental Design-Introduction to Solar Energy Systems Degree: $\quad$ AD, Environmental Design

Contact: Papousek, Connie

Students Taking or Completing Offering: Installer-

Residential (Solar System); Installer-Commercial (Solar

System); Solar Technician

\section{Solar Related Courses}

ED47, Alternate Energy Systems

Instructor:

House, Harold

Course Number:

31กR-01

Deparlment:

Environmental Design

Program or Curriculum: Envir. Des. - Intro. Sol.

Ener. Systems

\author{
Credits: 2 \\ Duration: $\quad 8$ Weeks, $3.0 \mathrm{hrs}$ per week \\ Contact Hours: $\quad 24$ \\ Classroom: \\ 24 \\ Topics Covered Extensively: Alternate Energy Sources; \\ Appropriate Technology; Intro. to Solar Energy; Photovol- \\ taics \\ Number of Times Taught: 3 \\ Average Enrollment: $\quad 15$ \\ Intro. to Solar Energy Systems \\ Instructor: House, Harold \\ Course Number: $\quad$ ED31 \\ Department: Environmental Design \\ Program or Curriculum: Envir. Des. - Intro. Sol. \\ Credits: \\ Ener. Systems \\ Duration: \\ Contact Hours: \\ 2 \\ Classroom: $\quad 48$ \\ Laboratory: $\quad 16$ \\ Topics Covered Extensively: Energy Storage; Heat and \\ Energy Transfer; Intro. to Solar Energy; Passive Solar \\ Technology; Solar System Components; Solar Home Con- \\ struction; Solar Collector Evaluation/Design; Solar \\ Systems Design; Space Heating; Space Cooling \\ Residential Energy Conservation \\ Instructor: House, Harold \\ Course Number: $\quad 3105-01$ \\ Department: Environmental Design \\ Program or Curriculum: Envir. Des. - Intro. Sol. \\ $\begin{array}{ll}\text { Credits: } & 2 \text { Ener. Systems } \\ \text { Duration: } & 3 \text { Weeks, } 8.0 \mathrm{hrs} \text { per week } \\ \text { Corltact Hours: } & 24\end{array}$ \\ $\begin{array}{cc}\text { Classroorl: } & 24 \\ \text { Topics Covered Extensively: Appropriate Technology; }\end{array}$ \\ Energy Conservation; Energy Storage; Heat and Energy \\ Transfer; Intro to Solar Energy; Marketing/Market Analysis: \\ Passive Solar Technology; Solar Home Construction: Solar \\ Systems Design; Domestic Hot Water; Space Heating; \\ Space Cooling \\ Number of Times Taught: 3 \\ Average Enrollment: $\quad 15$
}

Evergreen Valley College

[12452]

San Jose, California 95121

(408) 274-7900

Programs and Curricula

"Solar Technician

Students Taking or Completing Offering:

Solar Technician

Solar Related Courses

* Solar and Energy Seminar

"Solar House

"Two Courses on Solar

Program or Curriculum: "Solar Technician
[1219]

Long Beach, California 90808

(213) 420-4111

Programs and Curricula

*Air Conditioning and Refrigeration

Solar Related Courses

- Solar Segment

Department: Air Cond. \& Refrig.

Program or Curriculum: "Air Cond. \& Refrig. 
Modesto Junior College [1240]

Modesto, California 95350

(209) 526-2000

Programs and Curricula

External - Project Sunrise

Contact: Wilson, E. William

(209) $526-2000$

Solar Related Cuirses

Solar Energy Applications

Instructor:

Wilson, E. William

(209) $526-2000$

Course Number: - PS368

Department: : Dept. Engineering, Physicial Science and Mathematics

Program or Curriculum: External - Project Sunrise Credits: 3

Student Level: $\quad$ All Levels

Duration: $\quad 16$ Weeks, $3.0 \mathrm{hrs}$ per week

Contact Hours: . $\quad 48$

Classroom: ' 42

Laboratory:

6

Topics Covered Extensively: Alternate Energy Sources:

Energy Conservation; Energy Storage; Passive Solar

Tęrhnningy; Snlar System Comoonentș; Solar Economics;

Solar Home Construction: Domestic Hot Water; Space

Heating; Space Cooling; Wind Power, Small Systems

Number of Times Taught: 4

Average Enrollmenl: $\quad 35 \therefore$

\section{Monterey Peninsula College [1242] \\ Monterey, California 93940 \\ (408) 649-8000}

Programs and Curricula

Solar Collector Fabrication

$\begin{array}{lll}\text { Degree: } & & \text { Certificate-Solar Cullectors } \\ \text { Contact: } & \therefore & \text { Owen,Patrick } \\ & & (408) 649-1150\end{array}$

Solar Related Courses

Introduction to.Solar and Alternate Energy Sources

Iristructor: Dick Lee

Course Number: $\quad$ (408) 649

Department: Engineering

Program on Curricillum: Solar Collestor

Fabrication

Credits:

3

Student Level: All Levels

Duration:

Contact Hours:

18 weeks, 3 hrs per week

54

Topics Covered Extensively: Alternate Ënergy Sources;

Appropriate Technology; Energy Conversion; Energy

Storage; Passive Solar Technology; Photovoltaics; Solar

System Components; Solar Economics; Solar Collector

Evaluation/Design; Solar Systems Design; Solar Systems

Testing and Evaluation; Domestic Hot Water; Space Heating;

Space Cooling

Number of Times Taught: 3

Average Enrollment: $\quad 26$

\section{Mount San Antonio College [1245]}

Walnut, California 91789

(714) 598-2811

\section{Programs and Curricula}

Air Conditioning, Heating and Ventilation

Degree:

$A D$; Air Conditioning, Healing and Refrigeration

Contact:
Students Taking or Completing Offering

Electrician, Solar Technician, Installer-Residentia (Solar System), Installer-Commercial (Solar System). Plumber

\section{Solar Related Courses}

Solar and Alternate Energy Sources

$\begin{array}{ll}\text { Instructor: } & \text { Bormann, Jay } \\ & (714) 594-5611 \\ \text { Cuulse Nuritiei:: } & 70 \\ \text { Department: } & \text { Electronics } \\ \text { Program or Curriculum: } & \text { Air Cond., Heat., and Vent. } \\ \text { Credits: } & 3 \\ \text { Student Level: } & \text { Freshman or Sophomore } \\ \text { Duration: } & 18 \text { Weeks, 3.0 hrs per week } \\ \text { Contact Hours: } & 54 \\ \text { Classroom: } & 54\end{array}$

Topics Covered Extensively: Alternate Energy Sources; Appropriate Techlnology; Energy Conservation; Heat and Energy Transfer; Intro. to Solar Energy; Plumbing Techniques; Solar System Components; Solar Economics; Solar Systems Installation; Solar Systems Maintenance; Solar Systems Testing and Evaluation; Space Heating

Solar Energy Systems Installation

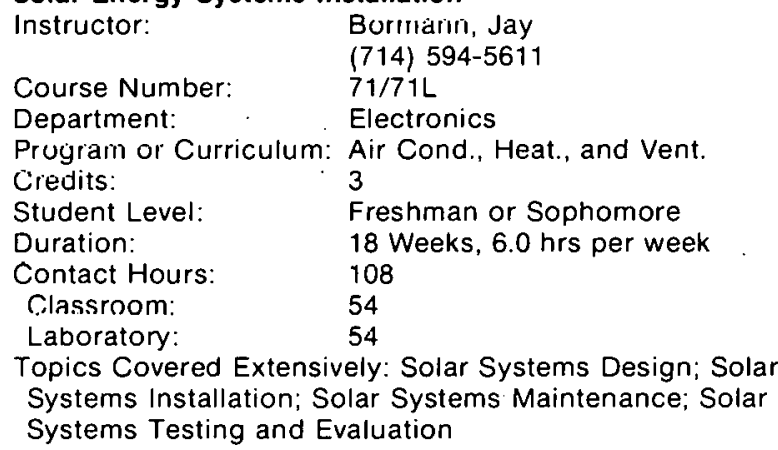

Office of Appropriate Technology [90530]

153010 th Street Sacramento, California 95814

Programs and Curricula

"Tralining Program for Insiallers

Contact: Jan Philbin (916) $445-1803$

Students Taking or Completing Offering

Inetallor-Residential (Solar System), Instalter-Commercial (Solar System)

Redwoods, College of the [1185]

Eureka, California 95501

(707) 443-8411

Solar Related Courses

Solar Heating A

Instructor: Mills, David

(707) $443-8411$

ENSC 20A

Uepartment: Eriv. Sci.

Credits:

1

Student Level: All levels

Duration: $\quad 12$ Weeks, $1.0 \mathrm{hrs}$ per week

Contact Hours: $\quad 12$ Classroom:

12

12

Topics Covered Extensively: Alternate Energy Sources: Energy Conservation; Energy Conversion; Energy Storage; Heat and Energy Transfer; Intro. to Solar Energy; Phuluvoltaics; Passive Solar Technology; Solar System Components; Solar Economics; Solar Home Construction: Solar Collector Evaluation/Design; Solar Systems Design; Solar Systems Installation; Sular Systems Testing and Evaluation; Domestic Hot Water; Swimming Pool Heating; Space Heating

Number of Times Taught: 1

Average Enrollmenl: $\quad 50$ 
Solar Heating $\mathbf{B}$

Instructor:

Mills, David

(707) 443-8411

Course Number

ENSC 20B

Department:

Env. Sci.

Credits:

Student Level:

All levels

Duration:

Contact Hours:

12 Weeks, $1.0 \mathrm{hrs}$ per week

12

12

Classroom:

Topics Covered Extensively: Alternate Energy Sources;

Energy Conservation; Energy Conversion; Energy

Storage; Heat and Energy Transfer; Intro. to Solar Energy;

Passive Solar Technology; Photovoltaics; Solar System

Components; Solar Economics; Solar Home Construction;

Solar Collector Evaluation/Design; Solar Systems Design;

Solar Systems Installation; Solar Systems Testing and

Evaluation; Domestic Hot Water; Swimming Pool Heating;

Space Heating

Number of Times Taught: 1

Average Enrollment: 50

Solar Heating $\mathbf{C}$

Instructor:

Mills, David

(707) 443-8411

Course Number:

ENSC 200

Department:

Env. Sci.

Credits:

1

All levels

Duration: $\quad 12$ Weeks, $1.0 \mathrm{hrs}$ per week

Contact Hours: $\quad 12$

Classroom:

12

Topics Covered Extensively: Alternate Energy Sources:

Energy Conservation; Energy Conversion; Energy

Storage; Heat and Energy Transfer; Intro. to Solar Energy;

Passive Solar Technology; Photovoltaics; Solar System

Components; Solar Economics; Solar Home Construction;

Solar Collector Evaluation/Design; Solar Systems Design;

Solar Systems Installations; Solar Systems Testing and

Evaluation; Domestic Hot Water; Swimming Pool Heating;

Space Heating

Number of Times Taught: 1

Average Enrollment: $\quad 50$

\section{San Diego Community College - City College San Diego, California 92101 [8895] (714) 238-1181}

\section{Programs and Curricula}

* Solar Energy, Maintenance and Technology

Degree:

AD, Advanced Degree

\section{Solar Related Courses}

"Two Courses on Solar Energy Maintenance, Technology Program or Curriculum: "Solar Ener. Maint. and Tech.

Topics Covered Extensively: Solar System Components;

Solar Economics; Solar Collector Evaluation/Design:

Solar Systems Installation; Solar Systems Maintenance;

Solar Systems Testing and Evaluation

\section{San Diego Community College - Evening College [7478]}

San Diego, California 92101

(714) $238-1 " 181$

\section{Programs and Curricula}

Air Conditioning, Heating, Refrigeration \& Solar Heatịng Technology

Degree:

$A D, O T H E R$, Air Conditioning \&

Heating, Refrigeration \& Solar

Contact:

Technology

Belker. Loren

(714) 238-1811

Students Taking or Completing Offering:

Solar Technician, Trade Specialty
Solar Related Courses

Advanced Solar Service Maintenance \& Technology

Instructor:

Faris, Theodore

Department

City Campus

Program or Curriculum: Air Cond. Heat.,

Credits: Refrig. and Sol. Tech.

Student Level: $\quad$ All levels

Duration: $\quad 18$ Weeks, $3.0 \mathrm{hrs}$ per week

Contact Hours: 54

Topics Covered Extensively: Appropriate Technology; Energy Conversion; Energy Storage; Heat and Energy Transfer; Intro. to Solar Energy; Plumbing Techniques; Solar System Components; Solar Home Construction; Solar Collector Evaluation/Design; Solar. Systems Design; Solar Systems Installation; Solar Systems Maintenance; Domestic Hot Water; Swimming Pool Heating: Space Heating

Number of Times Taught: 3

Air Conditioning, Heating, Refrigeration and Solar Energy Instructor:

Faris, Theodore

Course Number:

(714) 238-1181

Department: 201

Program or Curriculum: Air Cond., Heat., .

Credits: Refrig., and Sol. Tech.

Student Level

Duration:

Contact Hours:

Classroom:

Laboratory:

4

All levels

18 Weeks, $6.0 \mathrm{hrs}$ per week 108

54

54

Topics Covered Extensively: Appropriate Technology; Energy Conversion; Energy Storage; Heat and Energy Transfer; Intro. to Solar Energy; Plumbing Techniques; Solar System Components; Solar Home Construction; Solar Collector Evaluation/Design; Solar Systems Design; Solar Systems Installation; Solar Systems Maintenance; Domestic Hot Water; Swimming Pool Heating: Space Heating

Number of Times Taught: 3

Average Enrollment: $\quad 35$

Solar Service Maintenance and Technology

Instructor: Faris, Theodore

Course Number: 225

Department: City Campus

Program or Curriculum: Air Cond., Heat.,

Ċredits:

Student Level:

Duration:

Contact Hours: 3 Refrig., and Sol. Tech.

All levels

18 Weeks, $3.0 \mathrm{hrs}$ per week 54

Topics Covered Extensively: Appropriate Technology;

Energy Conversion; Energy Storage; Heat and Energy

Transler; Intro. to Solar Energy; Plumbing Techniques:

Solar System Components; Solar Home Construction;

Solar Collector Evaluation/Design; Solar Systems Design;

Solar Systems Installation; Solar Systems Maintenance

Domestic Hot Water; Swimming Pool Heating; Space

Heating

Number of Times Taught: 3

Average Enrollment: $\quad 35$

San Jose City College [1282]

San Jose, California 95128

(408) 298-2181

Programs and Curricula

"Solar Technician

Degree:

Contact

AD. Science

Students Taking or Completing Offering:

Solar Technician 
*Solar Technician

Degree:

OTHER, Certificate of Achievement

Contact

Herrick, Clyde/Upton, S

Students Taking or Completing Offering:

Solar Technician

\section{Solar Related Courses}

* Solar Energy - Industrial Applications

Instructor:

Upton, Si

Cunise NJumber: SOL 111

Department: Solar Technology

Program or Curriculum: "Sular Technician

Credits:

3

-Solar Energy - Residential Applications

Instructor: Upton, Si

Course Number: SOL 113

Department: Solar Technology

Program or Curriculum: "Solar Technology

Credits:

3

* Solar Photoelectric Conversion

Instructor: Upton, Si

Course Number: $\quad$ SOL 116

Department: Solar Technology

Program or Curriculum: "Solar Technician

Credits:

2

"Solar Theory

Instructor:

Upton, Si

Course Number: PHYSCl21

Program or Curriculum: "Solar Technician

Credits:

3

\section{Solar Technician Tralning Program - Office of Appropriate Technology [90340]. 1322 "O" Street Sacramento, California 95814 \\ Programs and Curricula \\ *Solar Technician Training Program \\ Contact: Trujillo, JoAnn \\ (916) 322-7190 \\ Students Taking or Completing Offering: \\ Solar Ter,hnirian}

\section{Solarcon}

[90490]

PO Box 14875

San Francisco, California 94114

\section{Solar Related Courses}

*Installers Workshop

Department:

(415) 648-2159

Karellen Educational

Services

Topics Covered Extensively: Solar Systems Installation

\section{COLORADO}

Colorado Technical College [10148] Colorado Springs, Colorado 80907 (303) 598-0200

Programs and Curricula

Solar Engineering Technology

Degree: $\quad B S, A D$, Applied Science

Contact: Christensen, Edward

(303) 598-0200

Students Taking or Completing offering:

Solar Engineer, Solar Technician
Solar Related Courses

Associate Seminar

Instructor:

Christensen, Edward

(303) 598-0200

Course Number: $\quad$ SOL 250

Department: Solar Engineering Technology

Program or Curriculum: Solar Engineering Technology

Credits:

Student Level: $\quad$ Freshman or Sophomore

Duration: $\quad 11$ Weeks, $1.0 \mathrm{hrs}$ per week

Contact Hours: $\quad 11$

Topics Covered Extensively: Alternate Energy Sources

Directed Practice

Instructor:

Christensen, Edward

(303) 598-0200

Course Number:

SOL 299

Department:

Solar Engineering Technology

Program or Curriculum: Solar Engineering Technology

Credits:

Duration:

Classroom:

Freshman or Sophomore

Number of Times Taught:14

Average Enrollment: 6

Introduction to Energy

Instructor:

Sabo, Julius $J$

(303) 598-0200

Course Number: · SOL 100

Department: Solar Engineering Technology

Program or Curriculum: Solar Engineering Technology

Credits:

Student Level: All levels

Duration: $\quad 11$ Weeks, $3.0 \mathrm{hrs}$ per week

Contact Hours: $\quad 33$

Classroom: 33

Topics Covered Extensively: Alternate Energy Sources;

Appropriate Technology

Number of Times Taught: 10

Averaye Enrullonint. 15

Solar Design I

Instructor:

Christensen, Edward

(303) 598-0200

Course Number: $\quad$ SOL 220

Dopartmont: Solar Engineering Teshnelegy

Program or Curriculum: Solar Engineering Technology

Credits:

Sluderil Level: $\quad$ Freshman or Sophomore

Duration: 11 Weeks, $6.0 \mathrm{hrs}$ per week

Contact Hours: $\quad 66$

Classroom: 22

Laboratory: 44

Topics Covered Extensively: Heat and Energy Transfer; Solar

System Components; Solar Collector Evaluation/Design;

Solar Systems Design: Space Heating

Number of Times Taught: 9

Average Enrollment: $\quad 8$

Solar Design II

Instructor:

Christensen, Edward

(303) 598-0200

Course Number: $\quad$ SOL 221

Department: Solar Engineering Technology

Program or Gurriculum; Solar Engineoring Tochno!ngy

Credits: 4

Student Level: $\quad$ Freshman or Sophomore

Duration: $\quad 11$ Weeks, $6.0 \mathrm{hrs}$ per week

Contact Hours: $\quad 66$

Classroom: 22

Laboratory: 44

Topics Covered Extensively: Solar System Components;

Solar Collector Evaluation/Design; Solar Systems Design;

Domestic Hot Water; Space Heating

Number of Times Taught: 9 
Denver, Red Rocks Campus,

Community College of

Golden, Colorado 80401

(303) 988-6160

Programs and Curricula

Solar Energy - Installation and Maintenance

Degree:

AD, OTHER, Solar EnergyInstallation and Maintenance

Contact: Hilton, Craig/Hilton, Robert (303) $988-6161$

Students Taking or Completing Offering:

Educator, Researcher, Installer-Residential (Solar System).

Plumber, Sheet Metal Worker

Solar Related Courses

Advance Solar Controls

Instructor:

Klima, John

(303) $988-6161$

Course Number:

SOM 236

Department: Industrial Occupations

Program or Curriculum: Solar Energy-Instal. and Main

Credits:

Student Level:

All levels

Duration:

Contact Hours:

15 Weeks, $4.0 \mathrm{hrs}$ per week

Classroom:

Laboratory:

60

30

Topics Covered Extensively: Solar System Components:

Solar Systems Testing and Evaluation; Domestic Hot

Water; Swimming Pool Heating; Space Heating

Number of Times Taught: 1

Average Enrollment: 25

Alternate Backup Systems for Solar Energy

Instructor:

Hilton, Craig

(303) 988-6161

SOM 238

Course Nurituet: Industrial Occupations

Department: Solar Energy-Instal. and Main. Credits: 3

Student Level:

Duration:

Contact Hours:

All levels

15 Weeks, $4.0 \mathrm{hrs}$ per week

Classroom:

Laboratory:

60

30

Topics Covered Extensively: Alternate Energy Sources

Basic Sheet Metal for Solar Energy

Instructor:

DuPriest, Don

(303) $988-6161$

Course Number:

SHM 100

Department: Industrial Occupations

Program or Curriculum: Solar Energy-Instal. and Main.

Credits:

Student Level:

3

Duration:

Contact Hours:

Classroom:

Laboratory:

All levels

15 Weeks, $4.0 \mathrm{hrs}$ per week

60

15

45

Topics Covered Extensively: Sheet Metal Techniques; Solar

System Components

Number of Times Taught: 3

Average Enrollment: 25

Basic Solar Controls

Instructor:

Hitz, Frank

(303) $988-6161$

Course Number:

SกM 235

Department

Industrial Occupations

Program or Curriculum: Solar Energy-Instal. and Main

Credits:

Student Level:

Duration:

All levels

Contact Hours: $\quad 60$

Classroom: $\quad 15$

Laboratory: $\quad 45$

Iopıcs Covered Extengively: Solar System Components;

Solar Systems Testing and Evaluation

Number of Times Taught: 2
Average Enrollment:

30

Basic Solar Systems

Instructor:

Course Number:

Hilton, Craig

(303) $988-6161$

SOM 220

Department:

Industrial Occupations

Program or Curriculum: Solar Energy-Inst. and Main.

Credits:

Student Level:

Duration:

Contact Hours:

All levels

Classroom:

Laboratory:

15 Weeks, $4.0 \mathrm{hrs}$ per week

Topics Covered Extensively: Alternate Energy Sources Energy Conservation: Intro. to Solar Energy; Plumbing Techniques; Solar Home Construction; Solar Systems Installation; Domestic Hot Water; Swimming Pool Heating; Space Heating

Number of Times Taught: 6

Average Enrollment: 20

Blueprint Reading For Construction Trades

Instructor: Feister, Clarence

Department: Industrial Div.

Program or Curriculum: Solar Energy-Instal. and Main.

Credits: 4

Student Level: $\quad$ All levels

Duration: $\quad 15$ Weeks, $4.0 \mathrm{hrs}$ per week

Contact Hours: $\quad 68$

Classroom: $\quad 45$

Laboratory: $\quad 23$

Number of Times Taught: 20

Average Enrollment: 20

Bricklaying For Construction Trades

Instructor: Gale, Bud

(303) 988-6161

Course Number: $\quad$ BRI 120

Department: Industrial Occupations

Program or Curriculum: Solar Energy-Instal. and Main.

Credits:

Student Level: All levels

Duration: $\quad 15$ Weeks, $4.0 \mathrm{hrs}$ per week

Contact Hours: $\quad 60$

Classroom: $\quad 15$

Laboratory: $\quad 45$

Number of Times Taught: 8

Average Enrollment: 20

Carpentry for Construction Trades

Instructor: Hinz, Tim

(303) $988-6161$

Course Number: $\quad$ CAR 120

Department: Industrial Occupations

Program or Curriculum: Solar Energy-Instal. and Main.

Credits: 3

Student Level. All levels

Duration: $\quad 15$ Weeks, $4.0 \mathrm{hrs}$ per week

Contact Hours: $\quad 60$

Classroom: $\quad 15$

Laboralory:

45

Topics Covered Extensively: Intro. to Solar Energy

Number of Times Taught: 8

Average Enrollment:

Domestic Hot Water

Instructor:

Course Number:

Department:

Hilton, Graig

(303) 988-6161

Program or Curriculum: Solar Energy-Instal. and Main.

Credits:

Student Level:

Duration:

Contact Hours:

Classroom:

Laboratory:

SOM 227

Topics Cov Domestic Hot Water 
Number of Times Taught: 4

Average Enrollment: 25

Hot Water Heating-Installation and Maintenance

Instructor: Hilton, Robert

(303) $988-6161$

PLU 206

Course Number Industrial Occupations

Department: Solar Energy-Instal. and Main.

Program or Curriculum:
Credils.

Duration:

All levels

15 Weeks, 4.0 hrs per week

Contact Hours:

60

Classroom:

Laboratory:

15

45

Topics Covered Extensively: Plumbing Techniques

Number of Times Taught:25

Average Enrollment: 25

Introduction to Photovoltaic and Wind Energy (303) 988-6161

Course Number: . SOM 239 Industrial Occupations

Department:

Program or Curriculum: Solar Energy-Instal. and Main.

Credits: 3

Student Level: $\quad$ All levels

Duration: 15

Contact Hours: $\quad 60$

Classroom: $\quad 30$

Liburatory: 30

Topics Covered Extensively: Alternate Energy Sources;

Photovoltaics; Solar Systems Installation; Elec'l Generation,

Small Scale; Wind Power, Small Systems

Orientation of Tools, Basic Plumbing, and Drawing

Instructor:

Hilton, Robert

(303) $988-6161$

Course Number:

PLU 100

Industrial Occupations

Program or Curriculum: Solar Energy-Instal. and Main.

Crendits: 3

Student Level:

All levels

Duration:

Contact Hours:

15 Weeks, 4.0 hrs per week

60

15

Laboratory

Topics Covered Extensively: Plumbing Techniques

Number of Times Taught: 25

Average Enrollment: 25

Passive Solar Systems

Instructor:

Shippee, Paul

(303) 988-6161

Course Number:

SOM 237

Industrial Occupations

Program or Curriculum: Solar Energy-Instal. and Main.

Credits: 3

Student Level:

"Duration:

Cuntact Hours:

All levels

15 Weeks, 4.0 hrs per week

60

30 .

30

Laboratory:

Topics Covered Extensively: Heat and Energy Transfer;

Passive Solar Technology; Solar Systems Design; Space

Heating

Number ol Times Taught: 1

Average Enrollment: 25

\section{Solar Engineering Technology I}

Instructor:

Haugseth, Larry

(303) 988-6161

SOM 221

Industrial Occupations

Course Number:

Department:

Solar Energy-Instal, and Main.

Program or Curriculum:

Student Level:

Duration:

Contact Hours:

Classroom:

All levels

15 Weeks, 4.5 hrs per week

68

45

23
Topics Covered Extensıvely: Solar Systems Design; Domestic Hot Water; Swimming Pool Heating; Space Heating

Number of Times Taught: 4

Average Enrollment: 25

Solar Engineering Technology II

Instructor: Dahl, Mike

$\begin{array}{ll}\text { Course Number: } & \text { (303) } 988-6161 \\ \text { Dopartment: } & \text { SOM } 222 \\ & \text { Indilstrial nrriupations }\end{array}$

Program or Curriculum: Solar Energy-Inst. and Main.

Credits:

Student Level: All levels

Duration: $\quad 15$ Weeks, $4.5 \mathrm{hrs}$ per week

Contact Hours: $\quad 68$

Classroom: 45

Laboratory: 23

Topics Covered Extensively: Solar Economics; Solar

Systems Design; Domestic Hot Water; Swimming Ponl

Heating; Space Heating; Space Cooling

Number of Times Taught: 3

Average Enrollment: 25

Solar Panel Arrays

Instructor:

Course Number: $\quad$ SOM $2 ? 6$

Hilton, Craig

(303) 988-6161

Department: Iridustrial Occupations

Program or Curriculum: Solar Energy-Instal. and Main

Credits:

Student Level: $\quad$ All levels

Duration:

Contact Hours:

Classroom

Laboratory:

15. Weeks, 4.0 hrs per week

60

15

4.5

Topics Covered Extensively: Solar System Components

Number of Times Taught: 6

Average Enrollment:

20

Solar Panel Installations

!nstructor:

Iilton, Craig

Course Number: $\quad$ SOM 229

Uepartment: Industrial Occupations

Program or Curriculum: Solar Energy-Instal. and Main.

Credits:

Student Level:

Duration:

Contact Hours:

Classroom:

l.aboratory

Topics Covered Extensively: Solar Home Construction; Solar Systems Installation

Solar System Design and Layout

Instructor: Hilton, Craig

Course Number: $\quad$ SOM 225

Department: Industrial Occupations

Program or Curriculum: Solar Energy-Instal. and Main.

Groditc: $\quad 3$

Student Lcvel: $\quad$ All levels

Duration:

15 Weeks, 4.0 hrs per week

Contact Hours: $\quad 60$

Classroom: $\quad 15$

Laboratory: 45

Topics Covered Extensively: Solar Collector Evaluation/

Design; Solar Systems Design

Number of I imes liaught: 6

Average Enrollment: 25

Solar System Maintenance

instructor:

Hilton, Craig

(303) $988-616$

Course Number: $\quad \because$ SOM 228

Department: Industrial Occupations

Program or Curriculum: Solar Energy-Instal. and Main.

Credits:

Student l.evel: All levels 
Duration:

Contact Hours:

15 Weeks, $4.0 \mathrm{hrs}$ per week

Classroom:

60

Laboratory:

15

45

Topics Covered Extensively: Solar System Components;

Solar Systems Maintenance

Number of Times Taught: 2

Average Enrollment: $\quad 25$

Water Piping Methods

Instructor:

Hilton, Robert

(303) $988-6161$

Course Number:

PLU 107

Industrial Occupations

Program or Curriculum: Solar Energy-Instal. and Main.

Credits: 3

Student Level: $\quad$ All levels

Duration: $\quad 15$ Weeks, $4.0 \mathrm{hrs}$ per week

Contact Hours:

Classroom:

60

Laboratory:

15

Topics Covered Extensively: Plumbing Techniques

Number of Times Taught: 25

Average Enroliment: 25

Otero Junior College [1362]

La Junta, Colorado 81050

(303) 384-4443

Programs and Curricula

Architecture Technology - Solar Heating Option

Degree: AD, Applied Science

Contacl:

Nilsen, E. W.

(303) 384-4443

Students 'Taking or Completing Offering:

Solar Technician, Trade Specialty

Solar Related Courses

Architecture Technology - Solar Heating Option

Instructor:

Nilsen, E. W.

(303) 384-4443

Department:

Construction \& Manufacturing

Program or Curriculum: Arch. Tech. - Solar

Heating Option

Student Level:

Freshman or Sophomore

Duration:

30 Weeks, 12.0 hirs per week 360

Topics Covered Extensively: Energy Storage; Intro. to Solar

Energy; Solar System Components; Solar Economics;

Solar Home Construction; Solar Collector Evaluation/

Design; Solar Systems Design; Solar Systems Installation;

Domestic Hot Water; Space Heating

Number of Times Taught: 0

\section{CONNECTICUT}

\section{None}

\section{DELAWARE}

Newcastle County Vocational Technical School
1417 Newport Rd.
[90370] Wilmington, Delaware 19804

Programs and Curricula

"Solar Heating of Buildings

Solar Related Courses

* Solar Heating of Buildings

Program or Curriculum: "Solar Heating of Buildings Contact Hours: 60

Topics Covered Extensively: Energy Conversion; Energy

Storage; Intro. to Solar Energy; Solar System

Components; Solar Economics; Solar Collector Evalua-

tion/Design; Solar Systems Design; Solar Systems

Installation; Solar Systems Maintenance; Solar Systems

Testing and Evaluation
DISTRICT OF COLUMBIA

National Training Fund [90360]

1900 "L" Street NW, Suite 405,

Washington, District of Columbia 20036

Programs and Curricula

"Sheet Metal - Apprentice, Journeyman

Contact:

Harrington, Mr. David

(202) 833-9543

FLORIDA

Brevard Community College [1470]

Cocoa, Florida 32922

(305) 632-1111

Programs and Curricula

Solar Engineering Technology

Degree: AD, Applied Science - Solar

$\begin{array}{ll} & \text { Engineering Techn } \\ \text { Contact: } & \text { Donnell, Nelson }\end{array}$

(305) 532-1111

Students Taking or Completing Offering:

Solar Technician

Florida Solar Energy Center [90100]

300 State Rd. 401

Cape Canaveral, Florida 32920

Solar Related Courses

"Short Courses, Workshops, Seminars

Gulf Coast Community College [1490]

Panama City, Florida 32401

(904) 769-1551

Programs and Curricula

Solar Energy Solar Systems

Degree:

Contact:

$A D$. Science

Jones, Robert C.

Solar Related Courses

Solar Energy

Instructor:

(904) $769-1551$

Course Number:

Department:

Stotz, Robert/ Jones, Robert (904) $769-1551$

CrM-1101

Tech. Ed. - A/C Heat. and Refrig.

Credits:

Student Level: All levels

Duration: $\quad 17$ Weeks, $3.0 \mathrm{hrs}$ per week

Contact Hours:

5

Topics Covered Extensively: Solar System Components;

Solar Home Construction; Solar Collector Evaluation/

Design

Number of Times Taught: 1

Average Enrollment: 24

Solar Systems

Instructor:

Courge Number:

Department:

Stotz, Robert/ Jones, Robert (904) $769-1551$

Credits:

Student Level:

Duration:

Contact Hours:

Solar Energy Solar Systems 3

All levels

17 Weeks, $3.0 \mathrm{hrs}$ per week

Covered Extensively: Heat and Energy Transfer;

Passive Solar Technology; Solar System Components;

Solar Economics; Solar Home Construction; Solar

Collector Evaluation/Design; Solar Systems Design;

Domestic Hot Water; Space Heating; Space Cooling

Number of Times Taught: 1

Average Enrollment: 
Miami-Dade Community College [1506]

Miami, Florida 33176

(305) 596-1211

Programs and Curricula

Air Conditioning Engineering Technology

Degree: $\quad A D$, Science

Contact: Succop, William

(3Us) $685-456 \overline{6}$

Students Taking or Completing Offering: Architect, InstallerResidential (Solar System), Installer-Commercial (Solar System), Solar Technician

\section{Solar Related Courses}

Solar Energy Fundamentals

Instructor:

Cleland, George

(305) 685-4206

Course Number:

ETM 2706

Department:

Air Conditioning Engineering

Technology

Program or Curriculum: Air Conditioning Engineering Technology

Gredits: 3

Student Level:

Muration:

rontact llours:

High School Graduate

15 Weeks, $3.0 \mathrm{hrs}$ per week 45

Topics Covered Extensively: Ënergy Conservation; Intro. 10 Solar Energy; Passive Solar Technology; Plumbing Techniques; Solar System Components; Solar Economics;

Solar Home Construction; Solar Systems Design; Solar Systems Installation; Domestic Hot Water; Swimming Pool Heating

Solar Energy Systems, Commercial

Instructor:

Cleland, George

(305) 685-4206

Course Number:

ETM 2758 C

Department:

Air Conditioning Engineering Technology

Program or Curriculum: Air Conditioning Engineering Technology

Credits: 3

Student Level:

Duration:

Cesilacl I lours:

High School Graduate

15 Weeks, $4 . U$ hris per week

Classroom:

Laboratory:

60

30

30

Topics Covered Extensively: Energy Conservation; Intro. to Solar Energy; Passive Solar Technology; Plumbing Techniques; Solar System Components; Solâr tconumiles, Solar Home Construction; Solar Systems Design; Solar Systems Installation; Domestic Hot Water; Swimming Pool Heating

Solar Energy Systems, Residential

Instructor:

Cleland, George

(305) 685-4206

Course Number:

ETM 2756C

Department:

Air Conditioning Engineering

Technology

Program or Curriculum: Air Conditioning Engineering Technology

Credits: 3

Sludent Level:

Duration:

Contact Hours:

Classroom:

Laboratory:

High School Graduate

15 Weeks, 4.0 hrs per week

60

30

30

Topics Covered Extensively: Energy Conservation; Intro. to Solar Energy; Passive Solar Technology; Plumbing Techniques; Solar System Components; Solar Economics;

Solar Home Construction; Solar Systems Design; Solar Systems Installaion; Domestic Hot Water; Swimming Pool Heating
Pensacola Junior College [1513]

Pensacola, Florida 32504

(904) 476-5410

Programs and Curricula

Solar Energy Technology

Degree:

AD, Energy Technology -

Contaot:

Certificate of Completion

Lnimery, Stanley

(904) $476-5410$

Solar Related Courses

Residential Design and Installation

Instructor:

Lowery, Stanley

(904) $476-5410$

Department: Industrial Technology

Program or Curriculum: Solar Energy Technology

Credits:

Student Level: $\quad$ Freshman or Sophomore

Duration: . 16 Weeks, 4.0 hrs per week

Contact Hours: $\quad 64$

Classroom: $\quad 32$

Laboratory: $\quad 32$

Tupics Covered Extensively: Energy Storage; Heat and Energy Transfer; Intro. to Solar Energy: Passive Solar Tochnology; Solar System r.nmnnnents; Solar Economire; Solar Home Construction: Solar Systems Design; Solar Systems Installation; Solar Systems Maintenance; Domestic Hot Water; Swimming Pool Heating; Space Heating; Space Cooling

South Florida Technical Institute [90020] 201 W. Sunrise Blvd.

Ft. Lauderdale, Florida 33311

(305) 764-3432

Programs and Curricula

Energy Conversion Systems

Contact: Linne, William L. (305) $764-3432$

Students Taking or Completing Offering: Mechanical or Electrical Contractor, Installer-Residential (Solar System). Trante Sperialty

Solar Related Courses

Air Conditioning, Refrigeration \& Major Appliances

Instructor:

Appleman, Louis

(305) 764-3432

Department:

Training

Program or Lurriculum: thepgy Converslun Byslemis

Student Level:

High School Graduale

Duration:

Contact Hours: 5 Weeks, $30.0 \mathrm{hrs}$ per week

Classroom:

Laboratory:

100

Topics Covered Extensively: Alternate Energy Sources; Appropriate Technology; Energy Conservation; Energy Conversion; Energy Storage; Heat and Energy T'ransfer; liatro. to Solar Lncrgy; Eolar Syetom Gompune!ts; Snlar Home Construction; Solar Collector Evaluation/Design; Solar Systems Design; Solar Systems Installation; Snlar Systems Maintenance; Solar Systems Testirig and Evaluation: Domestic Hot Water; Space Heating; Space Cooling Number of Times Taught: 3

Average Enrollment: $\quad 10$ 


\author{
GEORGIA \\ Dekalb Community College [1562] \\ Clarkston, Georgia 30021 \\ (404) 292-3994 \\ Programs and Curricula \\ Solar Heating \\ Degree: \\ Solar Heating \\ Contact: \\ Erickson, Gienn \\ (404) 292-1525 \\ Students Taking or Completing Offering: Installer- \\ Residential (Solar System), Solar Technician, Electrician, \\ Plumber. Sheet Metal Worker

\section{Solar Related Courses}

\section{Solar Heating} \\ Instructor: \\ Penland, William D. \\ (404) 292-1525 \\ Department: \\ Program or Curriculum: Solar Heating \\ Student Level: All levels \\ $\begin{array}{ll}\text { Duration: } & 14 \text { We } \\ \text { Contact Hours: } & 300\end{array}$ \\ Classroom: $\quad 200$ \\ Laboratory: $\quad 100$
}

HAWAII, IDAHO

None

\section{ILLINOIS} Illinois Eastern Community College - [1742]
Olney Central College

Olney, Illinois 62450

(618) 395-4351

Programs and Curricula

Construction Energy Program

$\begin{array}{ll}\text { Degree: } & \text { AD, Applied Science } \\ \text { Contact: } & \text { Marrs, Steve } \\ & \text { (618) 395-4351 }\end{array}$

Students Taking or Completing Offering: Installer-

Residential (Solar System), Solar Technician

\section{Solar Related Courses}

Energy Conservation Theory

$\begin{array}{ll}\text { Instructor: } & \text { Culver, Ray } \\ & (618) 395-4351 \\ \text { Coursc Numbcr: } & \text { SCI 121 } \\ \text { Department: } & \text { Physics } \\ \text { Program or Curriculum: Construction Energy Program } \\ \text { Credits: } & 3 \\ \text { Student Level: } & \text { Freshman or Sophomore } \\ \text { Duration: } & 12 \text { Weeks, 3.0 hrs per week } \\ \text { Contact Hours: } & 36 \\ \text { Classroom: } & 36\end{array}$

Túpics Cúvered Exleirisively: Alternate Energy Sourees; - Appropriate Technology; Biomass Conversion; Energy Conversion; Energy Storage; Heat and Energy Transfer; Intro. to Solar Energy; Passive Solar 'I echnology; Photovoltaics; Solar System Components; Solar Systems Design

Energy Systems in Construction Instructor:

Parish, William

(618) 395-4351

Course Number:

COT 172

Department: Construction Trades

Program or Ciırriculuım: Construction Energy Programm

Credits:

Student Level:

Freshman or Sophomore
Duration:

Contact Hours:

Classroom:

12 Weeks, $3.0 \mathrm{hrs}$ per week

36

Topics Covered Extensively: Alternate Energy Sources; Appropriate Technology; Biomass Conversion; Energy Conservation; Energy Conversion; Energy Storage; Heat and Energy Transfer; Intro. to Solar Energy; Passive Solar Technology; Photovoltaics; Solar System Components; Solar Home Construction; Solar Collector Evaluation/ Design; Solar Systems Design; Domestic Hot Water; Wind Power, Central Systems; Wind Power, Small Systems Number of Times Taught: 1

Average Enrollment: $\quad 30$

Gas and Arc Welding

Instructor:

Jausel, Russ

Course Number: $\quad$ (618) 395-4351

Department: Welding

Program or Curriculum: Construction Energy Program

Credits: 5

Student Level: $\quad$ All levels

Duration: $\quad 12$ Weeks, $8.0 \mathrm{hrs}$ per wek

Contact Hours: $\quad 96$

Classroom: 24

Laboratory: $\quad 72$

Number of Times Taught: 3

Average Enrollment: 12

Solar Store, Inc. [90030]

Box 841, Dept. bs

Peoria, Illinois 61652

(309) 673-6402

Programs and Curricula

* Solar Energy Education for Installers

Contact: Shanks, Diane

\section{INDIANA}

None

\section{IOWA}

Des Moines Area Community College [8735]

Ankeriy, lowa 50021

(515) 964-6200

Programs and Curricula

Solar Energy I and II

Degree:

Contact:

Adult Ed.

Rowe, Gordon N

(515) 964-6266

\section{Solar Related Courses}

\section{Man and Energy}

Insiructor:

Trumpy, Frank

Course Number:

Department:

Credits: 3 (515) 964-6292

Student Level: $\quad \checkmark \quad$ Freshman or Sophomore

Duration: $\quad 12$ Weeks, $3.0 \mathrm{hrs}$ per week

Contact Hours: $\quad 36$

Classroom: $\quad 36$

Topics Covered Extensively: Alternate Energy Sources Number of Times Taught: 9

Average Enrollment: 19

Solar Energy I - General Overview

Instructor: Sidles, Paul

Course Number: $\quad$ (516) 206-68A

Department: Adult Ed. 
Program or Curriculum: Solar Energy I and II

Student Level:

Duration:

Contact Hours:

Classroom:

All levels

10 Weeks, 3.0 hrs per week

Topics Covered Extensively: Energy Conservation; Intro. to

Solar Energy; Solar System Components; Solar

Economics; Solar Collector Evaluation/Design; Solar

Systems Design; Domestic Hot Water

Number of Times Taught: 7

Average Enrullment: 15

Solar Energy II - Air Systems

Instructor: Hummell, Myron

(515) 239-6900

Course Number: $\quad$ BLDG. 522

Department: Adult Ed.

Program or Curriculum: Solar Energy I and II

Student Level:" All levels

Duration: $\quad 10$ Weeks, $3.0 \mathrm{hrs}$ per week

Contact Hours: $\quad 30$

Classroom: 27

Topics Covered Extensively: Appropriate Technology;

Energy Cunservation; Energy Storage; Heat and Energy

Transfer; Sheet Metal Techniques; Solar System Compo-

nents; Solar 'Collector Evaluation/Design; Solar Systems

Design; Solar Systems Installation; Solar Systems Mainte-

nance; Space Heating

Number of Times Taught: 2

Average EnrolIment: $\quad 10$

\section{Muscatine Community College [1882] \\ Muscatine, lowa 52761 \\ (319) 263-8250 \\ Programs and Curricula \\ Solar Carpentry}

Degree:

BS, Industrial Education

Contact

Melander, Harry

(319) 263-8250

Students Taking or Completing Offering:

Installer-Residential (Solar System)

Use of Solar Energy - Homeowners, Builders

Degree: Certificate of Completion

Contact: . Ohlendorf, Vernon

(319) 263-8250

Students Taking or Completing Offering:

Do-it-yourself Homcowner

\section{Solar Related Courses}

\section{Solar Carpentry}

Instructor:

Melander, Harry

(319) 263-8250

Department

Trades

Program or Curricululum: Solar Carpentry

Credits:

Student Levei:

61

High School Graduate

Duration:

46 Weeks, $28.0 \mathrm{hrs}$ per week

Topics Covered Extensively: Energy Conservation; Energy

Conversion; Passive Solar Technology; Domestic Hot

Water

Number of Times Taught: 1

Average Enrollment:

10

Use of Solar Energy - Homeowners, Builders

Instructor:

Ohlendorf, Vernon

(319) 263-8250

Department:

Community Services-Cont. Ed.

Program or Curriculum: Use of Solar Energy -

Student Level: Homeowners, Builders

Duration:

All levels

Contact Hours:

10 Weeks, $3.0 \mathrm{hrs}$ per week

Classroom:

30

Laboratory:

20

10

Number of Times Taught: 2

Average Enrollment: 15
Scott Community College [4074]

Bettendorf, lowa 52722

(319) 359-7531

Programs and Curricula

* Solar Energetics Technology

Degree: $\quad A D$, Solar Energetics Technology (319) 359-7531

Students Taking or Complcting Offering: Architect,

Researcher, Installer-Residential (Solar System), Installer-

Commercial (Solar System), Solar lechnıcian

\section{Solar Related Courses}

"Courses: Installation, Repair - Heating, Refrigeration and Air Conditioning

Program or Curriculum: "Solar Energetics Technology

Topics Covered Extensively: Energy Storage; Photovoltaics; Plumbing Techniques; Sheet Metal Techniques; Solar System Components; Solar Collector Evaluation/ Design; Solar Systems Design; Solar Systems Installation; Solar Systems Maintenance; Solar Systems Testing and Evaluation; Domestic Hot Water; Process Heat, Industrial; Space Heating; Space Cooling

\section{Western lowa Tech [7316] \\ Sioux City, lowa 51102 \\ (712) $276-0380$}

Programs and Curricula

Solar Systems Technology

Degree:

AD, Applied Science in Solar

Systems Technology

Contact:

Chadwick, Richard

(712) 276-0380

Students Taking or Completing Offering: Installer-

Residential (Solar System), Installer-Commercial (Solar

System), Solar Technician, Other

Solar Related Courses

Blueprint Roading

Instructor:

Forsling, M. G.

(712) $276-0380$

Course Number: $\quad 274-3005$

Department: Trades \& Industry

Program or Curriculum: Solar Systems Technology

Credits:

4

Student Level: $\quad$ Freshman or Sophomore

Duration:

Contact Hours:

Classroom:

Laboratory:

12 Weeks, $5.0 \mathrm{hrs}$ per week

60

36

24

Topics Covered Extensively: Passive Solar Technology Plumbing Techniques; Sheet Metal Techniques; Solar System Components; Solar Home Construction; Sülar

Collector Evaluation/Design; Solar Systems Design; Elec'l

Generation, Small Scale; Space Heating

Average Enrollment: 11

Building Design for Solar Systems

Instructor: Forsling, M. G.

Course Number: $\quad 271-3010$

Department: $\quad$ Trades \& Industry

Program or Curriculum: Solar Systems Technology

Credits:

Student Level: $\quad$ Freshman or Sophomore

Duration: $\quad 12$ Weeks, 6.0 hrs per week

Contact Hours: $\quad 72$

Classroom: $\quad 24$

Laboratory: 48

Topics Covered Extensively: Energy Conservation; Energy

Storage: Passive Solar Technology; Solar Home

Construction

Average Enrollment: 
Integrated Solar Science II

$\begin{array}{ll}\text { Instructor: } & \text { Forsling, M. G. } \\ & (712) 276-0380 \\ \text { Course Number: } & 274-3006 \\ \text { Department: } & \text { Trades \& Industry } \\ \text { Program or Curriculum: } & \text { Solar Systems Technology } \\ \text { Credits: } & 5 \\ \text { Student Level: } & \text { Freshman or Sophomore } \\ \text { Duration: } & 12 \text { Weeks, 5.0 hrs per week } \\ \text { Contact Hours: } & 72 \\ \text { Classroom: } & 48 \\ \text { Laboratory: } & 24\end{array}$

Topics Covered Extensively: Energy Conversion; Elec'l

Generation, Small Scale; Space Heating; Space Cooling

Average Enrollment: $\quad 11$

Introduction to Solar Systems

Instructor: Forsling, M. G

(712) $276-0380$

Course Number: $\quad 274-3000$

Department: Trades \& Industry

Program or Curriculum; Solar Systems Technology

Credits:

3

Student Level:

Duration:

Contact Hours:

Freshman or Sophomore

12 Weeks, $3.0 \mathrm{hrs}$ per week

Classroom:

36

Average Erirullment: 11

Solar Feasabllity Cost Analysis

Instructor: Forsling, M. G

Course Number: (712) 276-0380

Department: Trades \& Industry

Program or Curriculum: Solar Systems Technology

Credits: 5

Student Level: $\quad$ Freshman or Sophomore

Duration: $\quad 12$ Weeks, 5.0 hrs per week

Contact Hours: $\quad 60$

Classroom: $\quad 60$

Topics Covered Extensively: Solar Economicics

Average Enrollment: 11

Solar Systems Applications I

Instructor:

Forsling, M. G.

(712) $276-0380$

Course Number: 274-3002

Department Trades \& Industry

Program or Curriculum: Solar Systems Technology

Credits:

9

Student Level:

Duration:

Contact Hours:

Freshman or Sophomore

12 Weeks, 13.0 hrs per week

Classroom

Laboratory:

156

60

Topics Covered Extensively: Energy Storage; Heat and

Energy Transfer: Plumhing Terhniques; Solar Collootor

Evaluation/Design

Average Enrollment:

11

Solar Systems Applications II

Instructor:

Forsling, M. G.

Course Number:

(712) $276-0380$

Department:

274-3007

Trades \& Industry

Prugram or Curriculum: Solar Systems Technology

Credits: $\quad 8$

Student Level: $\quad$ Freshman or Sophomore

Duration:

rontast Houre:

12 Weeks, $11.0 \mathrm{hrs}$ per week

132

Classroom: $\quad 60$

Laboratory: 72

Topics Covered Extensively: Energy Conservation; Energy Conversion; Energy Storage; Plumbing Techniques; Solar System Components; Solar Collector Evaluation/Design;

Solar Systems Testing and Evaluation; Space Heating

Average Enrollment: 11

Solar Systems Maintenance

Instructor:

Forsling, M. G

(712) $276-0380$
Course Number:

274-3013

Department:

Trades \& Industry

Program or Curriculum: Solar Systems Technology

Credits:

Student Level:

4

Duration:

Contact Hours:

Classroom:

Laboratory:

Freshman or Sophomore

12 Weeks, $6.0 \mathrm{hrs}$ per week

72

24

48

Topics Covered Extensively: Energy Storage; Solar System Components; Solar Systems Maintenance; Solar Systems Testing and Evaluation; Domestic Hot Water; Space Heating

Average Enrollment: $\quad 11$

\section{Systems Design Engineering}

Instructor:

Forsling, M. G.

Course Number:

(712) $276-0380$

Department:

274-3011

Program or Curriculum:
Credits:

Student Level:

Duration:

Contact Hours:

Classroom:

Laboratory:

Solar Systems Technolugy

6

Freshman or Sophomore

12 Weeks, 8.0 hrs per week 96

48

48

Topics Covered Extensively: Energy Storage; Heat and Energy Transfer; Solar Systems Design; Solar Systems Installation; Solar Systems Maintenance; Solar Systems

Testing and Evaluation

Average Enrollment:

11

\section{KANSAS}

Barton County Community Junior College

Great Bend, Kansas 67530

[4608]

(316) 792-2701

\section{Programs and Curricula}

Solar Energy Technology

Degree:

Contact:

AD, Applied Science

Greer, Neil

(316) 792-2701

Students Taking or Completing Offering: Trade Specialty

\section{Solar Related Courses}

Solar Energy and Applied Science I

Instructor

Greer, Neil

Course Number:

(316) $792-2701$

Department:

6900

Applied Sciences

Program or Curriculum: Solar Energy Technology

Credits:

Student Level:

3

Duration:

Contact Hours:

Freshman or Sophomore

17 Weeks, 6.0 hrs per week

Classroom:

102

Laboratory:

51

Topics Covered Extensively: Appropriate Technology; Heat and Energy Transier; Intro. to Solar Energy; Passive Solar Technology; Solar System Components; Solar Collector Evaluation/Design; Solar Systems Desiqn; Domestic Hot Water; Space Heating

Number of Times Taught: 1

Average Enrollment:

Kansas Technical Institute [4611]

Salina, Kansas 67401

(913) 825-0275

Programs and Curricula

Mechanical Engineering Technology - Solar Option 
Degree:

Contact:

$A D$, Science

Ashburll, M. H.

(913) 825-0275

Students Taking or Completing Offering: Solar Technician

\section{Solar Related Courses}

Solar System Design Technology.I

Instructor: Ashburn, $M$

(913) $825-0275$

Course Number: MT2832

Department: Mechanical Technology

Program or Curriculum: Mech. Engineering Tech. Solar Option

Credits: 2

Student Level:

Freshman or Sophomore

Duration:

Contact Hours: 16 Weeks, $4.0 \mathrm{hrs}$ per week 64

Classroom:

Laboratory:

16

48

Topics Covered Extensively: Energy Storage; Heat and Energy Transter: Intro. to Solar Energy; Passive Solar Technology; Plumbing Techniques; Solar System Components; Solar Economics; Solar Collector Evaluation/Design; Solar Systems Design; Solar Systems Installation; Solar Systems Testing and Evaluation vorrestle Hul Wuler; 3pace I leating

Solàr System Uesıgn Technulugy II

Instructor:

Ashburn, $M$.

(913) 825-0275

Course Number:

MT2844

Department: Mechanical Technology

Program or Curriculum: Mech. Engineering Tech. Solar Option

Credits: 4

Student Level:

Freshman or Sophomore

Duration: 16 Weeks, 8.0 hrs per week

Contact Hours:

Classroom:

Laboratory:

42

86

Topics Covered Extensively: Energy Storage; Heat and Energy Transfer; Intro. to Solar Energy; Passive Solar Technology; Plumbing Techniques; Solar System Components; Solar Economics; Solar Collector Evaluation/ Design; Solar Systems Design; Solar Systems Installation; Solar Šystems Testing and Evaluatıon; Uóméstic water Water; Space Heating

\section{KENTUCKY, LOUISIANA}

None

\section{MAINE}

\section{Cornerstones, Wing School of Shelter \\ Technology [90090] \\ 54 r. Iumberland St. \\ Brunswick, Maine 04011}

\section{Solar Related Courses}

Advanced New House

Course Number:

Duration:

B

Contact Hours:

3 Weeks, 35.0 hrs per week 105

Topics Covered Extensively: Passive Solar Technology;

Solar Home Construction

Passive Solar Building Design (207) $729-0540$

Course Number:

E

Duration:

Contact Hours:

1 Week, $35.0 \mathrm{hrs}$ per week 35

Topics Covered Extensively: Passive Solar Technology; Solar Home Construction

Passive Solar House Design \& Construction

Instructor:

Wing Charles

(207) $729-0540$
Course Number:

Credits:

Student Level:

Duration:

Contact Hours:

Classroom

Topics Covered Extensively: Energy Conservation; Heat and Energy Transfer; Intro. to Solar Energy; Passive Solar

Technology; Solar Home Construction

Number of Times Taught: 30

Average Enrollment: $\quad 30$

Retrofitting Existing Structures

Course Number:

Duration: (207) 729-0540

Contact Hours:

3 Weeks, $35.0 \mathrm{hrs}$ per week 105

Topics Covered Extensively: Alternate Energy Sources;

Passive Solar Technology; Solar Home Construction

\section{Solar Greenhouses}

Course Number: (207) 729-0540

Duration

Contact Hours:

1 Week, $35.0 \mathrm{hrs}$ per week 35

Topics Covered Extensively: Passive Solar Tcchnology;

Solar Home Construction

The Design Wnrkshnp

Instructor: Colburn, Gary

(207) 7200540

Tupics Covered Exlensively: Passive Solar Technology:

Solar Home Construction

Shelter Institute

58 Center Street

Bath, Maine 04530

Solar Related Courses

"Passive Solar Design

Instructor:

Hennin, Patsy

Duration:

(207) 443-7938

15 Weeks

Topics Covered Extensively: Passive Solar Technology;

Solar Home Construction

\section{MARYLAND}

RETS Technical Center [90050]

511 Russell Street

Baltimore, Maryland 21230

(301) 727-6863

Programs and Curricula

Retrlgeration Climate Control and Clean Air

Degree:

Refrigeration - Clımate Control clean Air

Contact:

Tickler, Earl M

(301) $727-6863$

Students Taking or Completing Offering: Installer-

Residential (Solar System), Solar Technician, Trade

Specialty

Solar Related Courses

Refrigeration - Climate Control - Clean Air

Instructor: Tickler, Earl M.

(301) 727-6863

Program or Curriculum: Refrig., Climate Control and

Student Level:

Clean Air

High School Graduate

Duration: $\quad 6$ Weeks, $30.0 \mathrm{hrs}$ per week

Contact Hours: $\quad 180$

Classroom:

Laboratory:

90

60

Topics Covered Extensively: Appropriate Techiology

Eneryy Conservation; Energy Storage; Heat and Energy 
Transfer; Intro. to Solar Energy; Passive Solar Technology; Plumbing Techniques; Solar Energy Policy Development; Solar System Components; Solar Economics; Solar Collector Evaluation/Design; Solat Systems Installation; Solar Systems Maintenance; Solar System Testing and Evaluation; Domestic Hot Water; Space Heating; Space Cooling

\section{MASSACHUSETTS}

\section{The Cambridge School - Weston Center For Open Education [90200] \\ Weston, Massachusetts \\ Solar Related Courses \\ *Adapting Heating Systems for Solar Use (617) 965-5428}

Topics Covered Extensively: Space Heating

*Advanced Studies in Solar Heating (617) $965-5428$

Topics Covered Extensively: Space Heating

*Basic Solar Heating (6.17) $965-5428$

Topics Covered Extensively: Space Heating

"Biomass for Energy

(617) $965-5428$

Topics Covered Extensively: Biomass Conversion

*Designing Your Own Solar System

(617) $965-5428$

Topics Covered Extensively: Solar System Components; Solar Collector Evaluation/Design; Solar System Design; Space Heating

"Photovoltaics

(617) $965-5428$

Topics Covered Extensively: Photovoltaics

*Power from the Sea

(617) $965-5428$

"Small Wind Mills

(617) $965-5428$

Topics Covered Extensively: Wind Power, Small Systems

* Solar Heating Added to Your House

(617) $965-5420$

*Solar Heating System Design

(617) $965-5428$

Topics Covered Extensively: Solar System Components; Solar Collector Evaluation/Design; Solar Systems Design; Domestic Hot Water; Space Heating

*Wind Machines

(617) $965-5420$

Topics Covored Extcnsively: Wiru Puwer, Cenlral Systems; Wind Power, Small Systems

\section{Cape Cod Community College [2168] \\ West Barnstable, Massachusetts \\ (617) 362-2131}

Programs and Curricula

Energy Systems Technology

$\begin{array}{ll}\text { Degree: } & \text { AD, Science } \\ \text { Contact: } & \text { Panitz, Ted } \\ & (617) 362-2131\end{array}$

Students Taking or Completing Offering: Solar Technician

\section{Solar Related Courses}

Energy Systems I-A Survey of Energy Alternatives

Instructor:

Panitz, Ted

(617) $362-2131$

Course Number:

TE 130

Department:

Industry Related Technology Pringram

Program or Curriculum: Energy Systems Technology Credits:

\author{
Student Level: $\quad$ Freshman or Sophomore \\ Duration: $\quad 15$ Weeks, 4.0 hrs per week \\ Contact Hours: $\quad 60$ \\ Classroom: $\quad 45$ \\ Laboratory: $\quad 15$ \\ Topics Covered Extensively: Alternate Energy Sources \\ Number of Times Taught: 3 \\ Average Enrollment: 15 \\ Energy Systems II - Solar Energy I \\ Instructor: \\ Panitz, Ted \\ Course Number: \\ (617) $362-2131$ \\ Department: \\ TE 131 \\ Industry Related Technologies \\ Credits: \\ Student Level: \\ Duration: \\ Contact Hours: \\ Classroom: \\ Laboratory: \\ Energy Systems Technology \\ 4 \\ Freshman or Sophomore \\ 15 Weeks, $4.0 \mathrm{hrs}$ per week \\ Topics Covered Extensively: Energy Storage; Heat and \\ Energy Transfer; Intro. to Solar Energy; Solar System \\ Components; Solar Collector Evaluation/Design; Solar \\ Systems Design; Domestic Hot Water; Space Heating \\ Number of Times Taught: 2 \\ Average Enrollment: $\quad 35$ \\ Energy Systems III - Solar Energy II \\ Instructor: Panitz, Ted \\ $\begin{array}{ll}\text { Course Number: } & \text { (617) } 362-2131\end{array}$ \\ Department: Industry Related Technologies \\ Program or Curriculum: Energy Systems Technology \\ Credits: 4 \\ Student Level: $\quad$ Freshman or Sophomore \\ Duration: $\quad 15$ Weeks, $1.0 \mathrm{hrs}$ pcr week \\ Contact Hours: $\quad 60$ \\ Classroom: $\quad 45$ \\ Laboratory: 15 \\ Tupics Covered Extensively: Heat and Energy Transfer; \\ Passive Solar Technology: Solar System Components; \\ Solar Economics; Solar Home Construction; Solar Collec- \\ tor Evaluation/Design; Solar Systems Design; Domestic \\ Hot Water; Space Heating \\ Number of Times Taught: 1 \\ Average Enrollment: 18
}

New England Fuel Institute [90230]

20 Summer St. Box 888

Watertown, Massachusetts 02172

\section{Solar Related Courses}

"Basic Snlar Heating Technology

Topics Covered Extensively: Space Heating

"Solar Installation and Maintenance

Instructor:

Tavino, R./ Taylor, R

(617) $924-1000$

Student Level:

All levels

Duration:

Contact Hours:

4 Weeks, $40.0 \mathrm{hrs}$ per week

Classroom:

Laboratory:

160

80

Topics Covered Extensively: Solar System Components; Solar Systems Installation; Solar Systems Maintenance; Domestic Hot Water; Space Heâtiñğ

\section{Northeast Institute of Industrial Technology} 41 Phillips St. [90060]

Boston, Massachusetts 02114

\section{Programs and Curricula}

\section{Installing Solar Water Heaters}

$\begin{array}{ll}\text { Degree. } & \text { Solar Water Systems } \\ \text { Contact: } & \text { Galvin, G. M. } \\ & \text { (617) 523-2813. }\end{array}$


Solar Related Courses

Installing Soular Water Heating

Instructor:

Smith, Robert $O . /$ Lannon, E. (617) 523-2813

Department: Air Conditioning, Refrig. Tech.

Program or Curriculum: Installing Solar Water Heaters

Student Level:

College Graduate

Duration:

15 Weeks, $2.0 \mathrm{hrs}$ per week

Contact Hoürs:

Classroom:

30

Number of Times Taught: 4

Average Enrollment: $\quad 30$

\section{Springfield Technical Community College Springfield, Massachusetts [8078] (413) 781-6470}

Programs and Curricula

* Solar Energy Option

Degree: $\quad A D$, Solar Energy

Contact: Murray, Carl

(413) $781-6470$

Solar Related Courses

* Conurses in Solar Technology

Department; Eng'r. Tech

Program or Curriculum: "Solar Energy Uption

Topics Covered Extensively: Alternate Energy Sources:

Appropriate Technology; Energy Conservation; Energy

Storage; Solar System Components; Solar Economics;

Solar Collector Evaluation/Design; Solar Systems Design;

Solar Systems Installation; Solar Systems Maintenance;

Domestic Hot Water; Space Heating

\section{MICHIGAN}

Charles S. Mott Community College [2261]

Flint, Michigan 48503

(313) $762-0200$

Programs and Curricula

Energy Technology

Degree:

AD, Applied Science, Alternate

Energy

Lidilie, Douglas :

Contact:

$$
\text { (313) 762-0278 }
$$

Students Taking or Completing Offering: Trade Specialty

\section{Solar Related Courses}

\section{Solar Heating and Cooling}

Instructor: Laine, Douglas E.

(616) $762-0278$

Course Number: PHYSCI-113

Dcpartment: Science and Mathematics

Piogram or Curriculum: Energy Technology

Credits: 2

Student Level: All levels

Duration: $\quad 16$ Weeks, $2.0 \mathrm{hrs}$ per week

Contact Hours: $\quad 32$

Classroom: . 32

Topics Covered Extensively: Energy Conservation; Energy Conversion; Energy Storage; Heat and Energy Transfer; Intro. to Solar Energy; Solar System Components; Solar Economics; Solar Collector Evaluation/Design; Solar

Systems Design; Solar Systems Installation; Solar Systems Maintenance; Solar Systems Testing and Evaluation

Number of Times Taught: 2

Average Enrollment: 20

\section{Ferris State College [2260]}

Big Rapids, Michigan 49307

(616) $796-9971$

Programs and Curricula

Refrigeration, Heating and Air Conditioning Technology
Degree

Contact:

$A D$, Applied Science in Refrig. Heating, and Air Conditioning

Shane, James B

(616) 796-9971

Students Taking or Completing Offering: Installer-

Commercial (Solar System), Installer-Residential (Solar System), Solar Technician, Trade Specialty

Solar Related Courses

Advanced Air Conditioning

Instructor:

Nott, Joe

(616) $796-9971$

Course Number: $\quad$ RHA 263

Department: Construction

Program or Curriculum: Refrig., Heating and Air Condi-

Credits: tioning Technology

Student Level:

Duration:

Contact Hours:

Classroom:

I ahoratory:

Freshman or Sophomore 10 Weeks, 20.0 hrs per week

Energy Conservation in Building Design

Instructor:

Kantor, Mel

(616) 796-9971

Course Number:

Departiment:

A-D 302

Program or Curriculum: Refrig., Heating and Air Condi-

Credits: tioning Technology

Student Level: $\quad$ All levels

Duration: $\quad 10$ Weeks, $3.0 \mathrm{hrs}$ per week

Contact Hours: $\quad 30$

Classroom: 30

Topics Covered Extensively: Energy Conservation; Passive Solar Technology; Solar Home Construction; Space Heating

Energy Use and Conservation

Instructor:

Erion, John

(616) $796-9971$

Course Number:

BCT 302

Department: Construction

Program or Curriculum: Refrig., Heating and Air Conditioning Technology

Credits 4

Student Level:

All levels

Duration:

Contact Hours:

10 Weeks, $5.0 \mathrm{hrs}$ per week

Clașsroom:

Laboratory:

50

30

20

Topics Covered Extensively: Alternate Energy Sources; Energy Conservation; Energy Conversion; Heat and Energy Transfer; Intro. to Solar Energy; Materials Research; Passive Solar Technology; Solar System Components; Solar Economics; Solar Systerns Installation; Domestic Hot Water; Space Heating

Heating

lissliuetor: Stovone, RusE

Course Number: (616) $796-9971$

Department: Construction

Program or Curriculum: Refrig., Heating, and Air Condi-

Credits: tioning Technology

Student Level:

Duration:

Contact Hours:

Classroom:

Laboratory:

9

Freshman or Sophomore 10 Weeks, $20.0 \mathrm{hrs}$ per week 200

50

150

Topics Covered Extensively: Heat and Energy Transfer; Intro. to Solar Energy; Solar System Components; Solar Economics; Solar Systems Maintenance; Space Heating Average Enrollment: ' 18

Summer Air Conditioning

Instructor:

Lawrence, Fred/Shaw, Dick

(616) $7966-9971$ 
Course Number:

RHA 261

Department:

Construction

Program or Curriculum: Refrig., Heating and Air Conditioning Technology

Credits: 9

Student Level:

Freshman or Sophomore

Duration:

Contact Hours:

10 Weeks, $20.0 \mathrm{hrs}$ per week

200

50

150

Laboratory:

Topics Covered Extensively: Plumbing Techniques; Solar

System Components; Solar Systems Installation; Solar

Systems Maintenance; Solar Systems Testing and Evaluation; Domestic Hot Water; Space Heating

Average Enrollment: 18

Grand Rapids Junior College [2267]

Grand Rapids, Michigan 49502

(616) 456-4895

Programs and Curricula

Architectural Drafting

Degree:

Contact:

$A D$, Architectural Drafting

Boyer, Don

Students Taking or Completing Offering: Architect

Heating, Ventilation, \& Air Conditioning

$\begin{array}{ll}\text { Degree: } & \text { AD, Heating, Ventilation, Air Cond. } \\ \text { Contact: } & \text { Boyer, Dori }\end{array}$

Solar Related Courses

Solar Systems - Collector Design and Construction

Instructor: Larson, L.

(616) 456-4860

Department: Technology

Program or Curriculum: Arch. Draft. and Heat., Vent., A/C

Student Level: $\quad$ Freshman or Sophomore

Duration: 16 Weeks, $4.0 \mathrm{hrs}$ per week

Contact Hours: $\quad 64$

Classroom: $\quad 16$

Laboratory: $\quad 32$

Topics Covered Extensively: Matcrials Research; Plumbing

Techniques; Solar System Components; Solar Collector

Evaluation/Design; Solar Systems Design; Solar Systems

Installation; Solar Systems Testing and Evaluation

Domestic Hot Water; Space Heating

Solar Dwelling Design Concepts

Instructor:

Larson. L.

(616) $456-4860$

Course Number:

TE 243

Department

Technology

Program or Curriculum: Arch. Draft. and Heat., Vent., A/C

Credits:

Student Level:

Freshman or Sophomore

Muration:

Contact Hours:

16 Weeks, 4.0 lirs per week

Classroom

64

32

32

Topics Covered Extensively: Appropriate Technology:

Energy Conservation; Energy Storage; Passive Solar

Technology; Solar Home Construclion; Domestic Hot

Water; Space Heating; Space Cooling

Solar Theory \& Design

Instructor:

Larson, L

(616) $456-4860$

Course Number:

TE 142

Department:

Technology

Program or Curriculum: Arch. Draft. and Heat., Vent., A/C

Credits:

2

Student Level:

Duration:

Contact Hours:

Classroom:

Laboratory:

Freshman or Sophomore

16 Weeks, $2.0 \mathrm{hrs}$ per week

32

28

Topics Cnvered Fxtensively: Enorgy Conversion; lintiu. lu

Solar Energy; Domestic Hot Water; Space Heating

Number of Times Taught:

Average Enrollment:
Lansing Community College [2278]

Lansing, Michigan 48901

(517) 373-7400

Solar Related Courses

*Alternate Sources of Energy

Course Number: ATG150

Department: Eng'r Tech.

Topics Covered Extensively: Alternate Energy Sources

"Building a Solar Furnace

Course Number:

ATG151

Department: Eng'r Tech

Topics Covered Extensively: Space Heating

* Building a Solar Water Heater

Course Number: ATG152

Department: Eng'r Tech.

Topics Covered Extensively: Domestic Hot Water

"Passive Solar Design

Course Number: AT211

Department: Eng'r Tech.

Topics Covered Extensively: Passive Solar Technology

"Passive Solar II

Course Number: AT215

Department: Eng'r Tech.

Topics Covered Extensively: Passive Solar Technology

"Principles of Solar Energy Collection

Course Number: AT201

Department: Eng'r Tech

Topics Covered Extensively: Solar System Components;

Solar Collector Evaluation/Design

*Residential Solar Heating System Design

Course Number: AT203

Department: Eng'r Tech. :

Topics Covered Extensively: Solar System Components;

Solar Home Curistruction; Solar Collector Evaluation/

Design; Solar Systems Design; Space Heating

* Solar Housing

Course Number:

AT200

Department:

Eng'r Ter.h.

* Solar Site Seminar

Course Number:

AT208

Department

Eng'r Tech

\section{MINNESOTA, MISSISSIPPI, MISSOURI, MONTANA}

None

NEBRASKA

Metropolitan Technical Community College

Omaha, Nebraska 68137

(402) $457-5100$

[12586]

Programs and Curricula

Solar Technical Training Program

Degree:

Solar Systems

Contact:

Kafka, Janies J.

(402) 457-5100

Students Taking or Completing Offering: Solar Technician

Solar Related Courses

Survey of Solar Energy

Instructor:

Reinmuth, Larry

(402) 457-5100

Department: Continuing Education

Program or Curriculum: Solar Technician Training Program

Student Level:

Duration:

Contact Hours:

All levels

Laborato

20

12 
Topics Covered Extensively: Alternate Energy Sources; Energy Conservation; Energy Storage; Heat and Energy Transfer; Intro. to Solar Energy; Passive Solar Technology; Photovoltaics; Solar System Components; Solar Collector Evaluation/Design; Solar Systems Design; Solar Systems Installation; Solar Systems Maintenance; Solar Systems Testing and Evaluation; Domestic Hot Water; Space Heating; Space Cooling; Wind Power, Small Systems

Number of Times laught: 3

Average Enroliment: 8

\section{NEVADA}

\section{Clark County Community College [10362]} Las Vegas, Nevada 89030

(702) 643-6060

\section{Programs and Curricula}

Solar Energy Technology

Degree:

AD, OT, Solar Energy Technology Applied Science

Contact:

Comarow, David (702) 843-6060

Students Taking or Completing Offering: Solar Technician, Shcet Metal Worker, Electrician, Plumber

Solar Relatod r.nirses

Advanced Solar Energy Technology

Instructor: Comarow, David

(702) 643-6060

Department:

Program or Curriculum: Solar Energy Technology

Credits: :

Student Level: $\quad$ Freshman or Sophomore

Duration: $\quad 15$ Weeks, $6.0 \mathrm{hrs}$ per week

Contact Hours: $\quad 90$

Classroom: $\quad 45$

Laboratory: 45

Topics Covered Extensively: Appropriate Technology; Energy Conservation; Energy Conversion; Energy Storage; Heat and Energy Transfer; Materials Research; Plumbing Techniques; Solar System Components; Solar System Components; Solar Economics; Solar Home Construction; Solar Law/Legislation; Solar Collector Evaluation/Design; Solar Systems Design; Solar Systems Installation; Solar Systems Maintenance; Solar Systems Testing and Evaluation; Domestic Hot Water; Swimming Pool Heating; Space Heating; Space Cooling

\section{Introduction to Solar Technology}

Instructor: Comarow, David

Course Number: (702) 643-6060

Department SOL 119

Program or Curriculum: Solar Energy Technology

Credits

Student Level:

Duration:

Contact Hours:

Classroom:

Laburatory:

Freshman or Suphomore

1.5 Weeks, $9.0 \mathrm{hrs}$ per week

13.

90

45

Topics Covered Extensively: Energy Conservation; Energy

Storage; Heat and Energy I ransfer; Intro. to Solar Energy;

Plumbing Techniques; Solar System Components; Solar

Economics; Solar Home Construction; Solar Collector

Evaluation/Design; Solar Systems Design; Solar Systems Installation; Solar Systems Maintenance; Solar Systems

Testing and Evaluation; Domestic Hot Water; Swimming

Pool Heating; Space Heating; Space Cooling

Passive Solar Heating and Cooling Technology

Instructor:

Comarow, David

(702) 643-6060

Course Number:

SOL 130

Department:

Science

Program or Curriculum: Solar Energy Technology

Credits:

3

Sludent Level:

Freshman or Sophomore
Duration:

Contact Hours:

15 Weeks, $3.0 \mathrm{hrs}$ per week

Classroom:

45

Topics Covered Extensively: Energy Storage; Heat and Energy Transfer; Passive Solar Technology; Solar Economics; Solar Home Construction; Space Heating; Space Cooling

Practicum in Solar Technology

Instructor: Comarow, David

Course Number: $\quad$ SOL 1210

Department: Science

Program or Curriculum: Solar Energy Technology

Credits: 3

Student Level: $\quad$ Freshman or Sophomore

Duration: $\quad 15$ Weeks, $9.0 \mathrm{hrs}$ per week

Contact Hours: 135

Topics Covered Extensively: Domestic Hot Water; Swimming Pool Heating; Process Heat, Industrial, Space

Heating; Space Cooling

Solar Energy Technology - Home Owner

Instructor: Comarow, David

(702) 643-6060

Course Number: $\quad$ ENV 1183

Department: Science

Program ur Gurriculum Snlar Fnergy Tcchnologr

Student I avel. All livels.

Duration: $\quad 1$ Week, $15.0 \mathrm{hrs}$ per week

Contact Hours: $\quad 15$

Classroom: 15

Topics Covered Exterısively: Alternate Energy Sources;

Appropriate Technology; Energy Conservation; Energy

Conversion; Energy Storage; Heat and Energy Transfer; Intro, to Solar Energy; Passive Solar Technoloqv;

Plumbing Techniques; Solar System Componerıls; Solar Economics; Solar Home Construction; Solar Collector

Evaluation/Design; Solar Systems Design; Solar Systems Installation; Solar Systems Maintenance; Solar Systems

Testing and Evaluation; Domestic Hot Water; Swimming

Pool Heating; Space Heating; Space Coolıng

Number of Times Taught: 7

Average Enrollment: 100

\section{NEW HAMPSHIRE}

\section{New Hampshire Vocational Technical College - Manchester [2582]}

Manchester, New Hampshire

(603) 668-6706

Programs and Curricula

Solar Energy Certificate Program

Degree: Solar Energy

Contact: Magnon, David

(603) 668-6706

Students Taking or Completing Offering: Educator, Do-it-yourself Homeuwrier

\section{Solar Related Courses}

Energy Conservation - Principles

Instructor: Magnon, David

(603) 668-6706

Course Number: M941EV

Department: Evening

Program or Curriculum: Solar Energy Certificate Program Credits: 3

Student Level: $\quad$ All levels

Duration: $\quad 12$ Weeks, $3.0 \mathrm{hrs}$ per week

Contact Hours:

36

Topics Covered Extensively: Energy Conservation

Number of Times Taught: 1

Average Enrollment: $\quad .20$

Energy Survey \& Alternative Systems

Instructor: Magnon, David

Course Number: $\quad$ M940EV 
Department:

Evening

Program or Curriculum:

Credits:

Solar Energy Certificate Program

Student Level:

Duration:

All levels

Contact Hours:

12 Weeks, $3.0 \mathrm{hrs}$ per week 36

Topics Covered Extensively: Alternate Energy Sources;

Appropriate Technology; Energy Conservation; Intro. to

Solar Energy; Passive Solar Technology

Number of Times Taught: 1

Average Enrollment: 20

Principles of Solar Design

Instructor:

Magnon, David

(603) 668-6706

Course Number: $\quad$ M943EV

Department: Evening Extension

Program or Curriculum: Solar Energy Certificate Program

Credits: 3

Student Level: $\quad$ All levels

Duration: $\quad 12$ Weeks, $3.0 \mathrm{hrs}$ per week

Contact Hours: $\quad 36$

Topics Covered Extensively: Solar Home Construction;

Solar Collector Evaluation/Design; Solar Systems Design

Solar Construction \& Installation Technology

Instructor: Magnon, David

(603) 668-6706

Course Number: $\quad$ M944EV

Department: Evening Extension

Program or Curriculum: Solar Energy Certificate Program

Credits:

Student Level:

Duration:

Contact Hours:

12 Weeks, $4.0 \mathrm{hrs}$ per week

Topics Covered Extensively: Solar Collector Evaluation/

Design; Solar Systems Design; Solar Systems Installation;

Solar Systems Maintenance

$\begin{array}{ll}\begin{array}{l}\text { Solar Energy - a Prime Energy Resource } \\ \text { Instructor: }\end{array} & \begin{array}{l}\text { Magnon, David } \\ \text { (603) 668-6706 }\end{array} \\ \text { Course Number: } & \text { M942EV } \\ \text { Department: } & \text { Evening Extension } \\ \text { Program or Curriculum: Solar Energy Certificate Program } \\ \text { Credits: } & 4 \\ \text { Student Level; } & \text { All levels } \\ \text { Duration: } & 12 \text { Weeks, } 4.0 \text { hrs per week } \\ \text { Contact Hours: } & 48\end{array}$

Topics Covered Extensively: Alternate Energy Sources;

Heat and Energy Transfer; Intro. to Solar Energy; Passive Solar Technology; Solar System Components; Solar

Collector Evaluation/Design; Space Heating

Solar Heating Systems

Instructor:

Byrne, $E$

Course Number: $\quad 404$

Department: HVAr.

Credits:

Student Level:

4

All levels

Duration

Contact Hours:

12 Weeks, $4.0 \mathrm{hrs}$ per week 48

Topics Covered Extensively: Plumbing Techniques; Sola Systems Installation; Solar Systems Maintenance; Solar Systerris Testing and Evaluation; Domestic Hot Water; Space Heating

Number of Times Taught: 4

Average Enrollment: 25

Solar Seminar - Integrated Projects

Instructor:

Magnon, David

Course Number:

(603) 668-6706

Department:

M945EV

Evening Extension

Program or Curriculum: Solar Energy Certificate Program

Credits:

Student Level:

Duration:

Contact Hours:
All levels

12 Weeks, $3.0 \mathrm{hrs}$ per week

36
NEW JERSEY

Essex County Technical Careers Center [90390]

91 West Market St.

Newark, New Jersey

Programs and Curricula

*Day Program - Solar Heating Systems

Degree: Certificate

*Night Program - Solar Heating Systems

Degree: Certificate

Solar Related Courses

"Solar Heating Systems -

(Day Course)

Department: Adult Education

Program or Curriculum: "Day Program - So. Heat. Systems

Contact Hours: 300

Topics Covered Extensively: Solar System Components;

Solar Collector Evaluation/Design; Solar Systems Design;

Solar Systems Installation; Solar Systems Maintenance;

Durnestic Hot Water; Space Heating; Space Cooling

* Solar Heating Systems - (Night Course)

Department: Adult Education

Program or Curriculum: "Night Program - Sol. Heat. Systems

Contact Hours: 120

Topics Covered Extensively: Solar System Components; Solar Collector Evaluation/Design; Solar Systems Design; Solar Systems Installation; Solar Systems Maintenance;

Domestic Hot Water; Space Heating; Space Cooling

\section{Mercer County Area Vocational \\ Technical Schools [90560] \\ 1085 Old Trenton Rd. \\ Trenton, New Jersey 08690}

Solar Related Courses

*Installing Solar Heating \& Cooling

Department: Plumbing, Heating, and Refrig.

Topics Covered Extensively: Plumbing Techniques; Solar System Components; Solar Systems Installation; Domestic Hot Water; Space Heating; Space Cooling

\section{Ocean County Vocational Technical Schools Route 571 \\ [90380]}

Jackson, New Jersey 08527

Programs and Curricula

"Solar Energy Theory-Heating, Ventilation, Air

Conditioning Technology

Degree:

Evening Sr.hnol rertifir.ate

\section{Solar Related Courses}

*Solar Energy Theory-Heating, Ventilation and

Air Conditioning

Department:

Evening School

Program or Curriculum: "Sol. Ener. Theory - Heat., Vent.,

Student Level:

A/C Tech

Duration:

All levels

Topics Covered Extensively: Solar System Components:

Solar Collector Evaluation/Design; Solar Systems Design; Domestic Hot Water; Space Heating

"Solar Energy Workshop

Department:

Student Level:

Evening School

Topics Covered Extensively: Solar System Components:

Solar Economics; Solar Collector Evaluation/Design;

Solar Systems Design; Solar Systems Installation; Domestic Hot Water 
Southern New Jersey OIC [90070]

Camden, New Jersey

Programs and Curricula

Solar Energy Unit Installer Program

Degree: $\quad$ Completion Certificate

Contact: Keene, Joseph P.

(609) -944-2545

Studonte Taking or rinmpleting ()ffering:

Installer-Residential (Solar System)

Solar Related Courses

Solar Energy Installer

Instructor: Keene, Joseph P

(609) 966-2545

Program or Curriculum: Solar Energy Unit Installer Program

Studenl Level:

High School Graduate

Duration:

26 Weeks, $5.0 \mathrm{hrs}$ per week 130

Topics Covered Extensively: Appropriate Technology;

Biomass Conversion; Energy Conservation; Energy Conversion; Energy Storage; Heat and Energy Transfer; Intro. to Solar Energy; Passive Solar Technology; Plumbing Techniques; Solar System Components; Solar Systems Installation; Domestic Hot Water; Swimming Pool Heating; Space Heating

\section{NEW MEXICO}

None

\section{NEW Y YORK}

Adirondack Community College [2860]

Glens Falls, New York 12801

(518) $793-4491$

Programs and Curricula

Seminar in Solar Energy

$\begin{array}{ll}\text { Degree: } & \text { Heating Certificate } \\ \text { Contact: } & \text { Harrington, Charles }\end{array}$

Contact: $\quad$ (518) 747-0274

Students Taking or Completing Offering:

Solar Techniçian

Solar Related Courses

Seminar in Solar Energy

Instructor:

Harrington, Charles

Course Number: (518) 747-0274

Department: TECH 191

Occ. Ed.

Program or Curriculum: Seminar in Solar Energy

Credits: 3

Student Level:

All levels

Duration:

Contact Hours:

17 Weeks, $6.0 \mathrm{hrs}$ per week

102

Classroom:

51

Laboratory:

51

Topics Covered Extensively: Energy Storage; Heat and

Energy Transfer; Intro. to Solar Energy; Passive Solar

Technology; Solar System Components; Solar Economics;

Solar Home Construction; Solar Collector Evaluation/

Design; Solar Systems Design; Solar Systems Instaiialiull;

Solar Systems Maintenance; Solar Systems Testing and

Evaluation; Domestic Hot Water; Space Heating;

Space Cooling

Number of Times Taught: 5

Average Enrollment: $\quad 25$

Cayuga County Community College [2861] Auburn, New York 13021

(315) 253-7345

Programs and Curricula

Solar Energy Technology

Degree:

Solar Energy Technology

Contact:

Komanecky, William

(315) 253-7345
Students Taking or Completing Offering:

Do-it-yourself Homeowner, Electrician, Plumber

Solar Related Courses

Solar Heating Energy

Instructor:

Simkin, Robert

(315) 364-8065

Department: Science

Program or Curriculum: Solar Energy Tectinology

Crcdits:

Student Level: $\quad$ All levels

Duration: 5 Weeks, 3.0 hrs per week

Contact Hours: $\quad 15$

Classroom: 15

Topics Covered Extensively: Energy Storage; Heat and

Energy Transfer; Intro to Solar Energy; Passive Solar Tech-

nology; Solar System Components; Space Heating

Number of Times Taught: 2

Average Enrollment: 23

CUNY New York City Community College [2696] Brooklyn, New York 11201 (212) 643-4033

Prngrams and Curricula

Environmental Control Technology

$\begin{array}{ll}\text { Degree: } & \text { AD, Applied Science } \\ \text { Contact: } & \text { Lomask, Samuel }\end{array}$

(212) 962-0407

Solar Related Courses

Environmental Design I

Instructor:

Farkas, Stanley

(212) 239-1662

Course Number: $\quad$ EC 110

Department: . Environmental Control Technology

Program or Curriculum: Environmental Control Technology

Credits: 3

Student Level: $\quad$ Freshman or Suphomore

Duration: $\quad 15$ Weeks, 3.0 hrs per week

Contact Hours: $\quad 45$

Classroom: $\quad 45$

Topics Covered Extensively: Energy Conservation; Heat

and Eneroy Transfer

Number of Times Taught: 70

Average Enrollment: 25 .

Environmental Design Laboratory

Instructor: Farkas, Stanley

(212) 239-1662

Course Number: $\quad$ EC 111

Department: Erivironmental Control Technology

Program or Curriculum: Environmental Control Technology

Credits: 1

Student Level: . Freshman or Sophomore

Duration: $\quad 15$ Weeks, $3.0 \mathrm{hrs}$ per week

Contact Hours: $\quad 45$

Classroom: 3

Topics Covered Extensively: Plumbing Techniques

Average Enrollment: 20

Environmental System nẹsign

Instructor: $\quad$ Finger, A.

(212) $238-1658$

Course Number: $\quad$ EC 430

Department: Environmental Control Technology

Program or Curriculum: Environmental Control Technology

Credits: $\quad .4$

Student Level: $\quad$ Freshman or Sophomore

Duration: $\quad 15$ Weeks, $6.0 \mathrm{hrs}$ per week

Contact Hours: $\quad 90$

Classroom: $\quad 30$

Laboratory: $\quad 60$

Number of Times Taught:16

Average Enrollment: 18

Hydronic Systems Design

Instructor:

Pita, Edward

(212) 239-1662 


\author{
Course Number: \\ EC 220 \\ Department: \\ Environmental Control Technology \\ Program or Curriculum: Environmental Control Technology \\ Credits: \\ 3 \\ Student Level: \\ High School Graduate \\ 15 Weeks, 4.0 hrs per week \\ Contact Hours: \\ 60 \\ 40 \\ Classroom: \\ ly: Heat and Energy Transfer \\ Topics Covered Extensively:
Number of Times Taught: 24 \\ Average Enrollment: 25

\section{Refrigeration II} \\ Instructor: \\ Lomask, Samuel \\ (212) 239-1696 \\ Course Number: \\ EC 410 \\ Department: \\ Environmental Control Technology \\ Program or Curriculum: Environmental Control Technology \\ Credits: \\ 2 \\ Student Level: \\ Junior or Senior \\ Duration: \\ 15 Weeks, $2.0 \mathrm{hrs}$ per week \\ 30 \\ Topics Covered Extensively: Energy Conservation: Energy \\ Storage; Heat and Energy Transfer \\ Number of Times Taught: 16 \\ Average Enrollment: $\quad 25$
}

\section{Mohawk Valley Community College [2871] \\ Utica, New York 13501 \\ (315) $792-5500$ \\ Programs and Curricula \\ Solar Energy Technology \\ Degree: \\ Contact: \\ Solar Energy Technology \\ Dunning, Francis \\ (315) $792-5514$}

\section{Solar Related Courses}

$\begin{array}{ll}\begin{array}{l}\text { Solar Energy I - Energy \& Energy Construction } \\ \text { Instructor: }\end{array} & \begin{array}{l}\text { Dunning, Francis } \\ (315) 792-5514\end{array} \\ & \text { CC } 530 \\ \text { Course Number: } & \text { Physics and Engineering Science } \\ \text { Department: } & 3 \\ \text { Program or Curriculum: Solar Energy Technology } \\ \text { Credits: } & \text { All levels } \\ \text { Student Level: } & 10 \text { Weeks, } 3.0 \text { hrs per week } \\ \text { Duration: } & 30 \\ \text { Contact Hours: } & 30\end{array}$

Topics Covered Extensively: Appropriate Technology;

Energy Conservation; Energy Storage; Heat and Energy

Transfer; Intro. to Solar Energy; Solar System Components;

Solar Economics; Solar Systems Installation; Domestic

Hot Water; Space Heating

Number of Times Taught: 5

Averaye Enrullinent: $\quad 30$

Solar Energy II (Installation)

Instructor:

Dunning, Francis

(315) $792-5514$

Course Number:

D2986

Department:

Physics

Program or Curriculum: Solar Energy Technology

Credits:

Student Level:

Duration:

Contact Hours:

Classroom

Laboratory:

Freshman or Sophomore

10 Weeks, $5.0 \mathrm{hrs}$ per week

50

30

Topics Covered Extensively: Appropriate Technology;

Energy Storage; Heat and Energy Transfer; Intro. to Solar

Energy; Plumbing Techniques; Solar System Components;

Solar Eronnomics: Solar Home Construction; Solar Systems

Design; Solar Systems Installation; Solar Systems Main-

tenance; Solar Systems Testing and Evaluation; Domestic

Hot Water; Space Heating

Number of Timoc Taught: 1

Averảge tnrollment: $\quad 30$
Solar III (Solar Energy System Design and Analysis)

Instructor:

Dunning, Francis

(315) 792-5514

Department:

Physics

Program or Curriculum: Solar Energy Technology

Credits:

Student Level:

4

Freshman or Sophomore

Duration:

Contact Hours:

10 Weeks, 5.0 hours per week

50

30

Classroom:

Laboratory:

20

Topics Covered Extensively: Appropriate Technology:

Energy Conversion; Energy Storage; Heat and Energy

Transfer; Materials Research; Plumbing Techniques; Sheet

Metal Techniques; Solar System Components; Solar

Economics: Solar Home Construction; Solar Collector

Evaluation/Design; Solar Systems Design; Solar Systems

Installation; Solar Systems Testing and Evaluation;

Domestic Hot Water; Swimming Pool Heating; Space

Heating; Space Cooling

Solar IV (Alternate Energy Sources)

Instructor: Dunning, Francis

(315) $792-5514$

Physics

Program or Curriculum: Solar Energy Technology

Credits:

Student Level:

Duration:

Contact Hours:

Classroom:

Laboralory:

4

Freshman or Sophomore

10 Weeks, $5.0 \mathrm{hrs}$ per week

Topics Covered Extensively: Alternate Energy Sources;

Energy Conservation; Energy Conversion; Intro. to Solar

Energy Passive Solar Technology; Photovoltaics; Solar

Economics; Solar Law/Legislation; Process Heat, Agricul-

tural; Process Heat. Industrial; Wind Power;

Central Systems; Wind Power, Small Systems

\section{Solar System Fabrication}

Instructor:

Dunning, Francis

Department

(315) $792-5514$

or Curriculum:

Student Level:

Duration

Contact Hours:

Classroom:

Laboratory:

Physics

Topics Covered Extensively: Appropriate Technology:

Plumbing Techniques; Sheet Metal Techniques; Solar

Systems Installation; Solar Systems Maintenance; Solar

Systems Testing and Evaluation; Domestic Hot Water;

Space Heating; Space Cooling

Solar System Fabrication II

Instructor:

Dunning, Francis

(315) $792-5514$

Department: Physics

Program or Curriculum: Solar Energy Technology

Credits:

4

Student Level:

Freshman or Sophomore

Duration:

Contact Hours:

10 Weeks, $7.0 \mathrm{hrs}$ per week

Classroom:

Laboratory:

10

Topics Covered Extensively: Appropriate Technology; Plumbing lechniques; Sheet Metal Techniques: Solar Systems Installation; Solar Systems Maintenance; Solar Systems Testing and Evaluation; Domestic Hot Water:

Space Heating; Space Cooling 
SUNY Agricultural and Technical College - Delhi Delhi, New York 13753

(607) $746-4111$

Programs and Curricula

Construction Technology/Civil Technology

Degree: Ad, Applied Science

Contact: Duncan, George

(607) 746-4225

Students Taking or Completing Offering:

Mechanical or Electrical Contractor, Contractor, Other

\section{Solar Related Courses}

\section{General Chemistry}

Instructor:

Onasch, Frederick
(607) $746-4377$

9512

Course Number:

Physical Scienccs

Department:

Construction Tech./Civil Tech.

Program or Curriculum.
Credits:

Student Level:

Duration:

Contact Hours:

Classroom:

Laboratory:

4 Freshman or Sophomore

15 Weeks, $5.0 \mathrm{hrs}$ per week

75

30

45

Topics Covered Extensively: Alternate Lnergy Sources;; Appropriate Technology; Biomass Conversion; Energy Conversion; Energy Storage; Heat and Energy Transfer; Materials Research; Photovoltaics

General Chemistry 9513

Instructor:

Onasch, Frederick

(607) $746-4377$

9513

Course Number:

Physical Sciences

Departiment:

Construction Tech./Civil Tech.

Credits:

4

Student Level:

Duration:

Contact Hours:

Classroom:

Laboratory:

Freshman or Sophomore

15 Weeks, $5.0 \mathrm{hrs}$ per week

75

30

Topics Covered Extensively: Alternate Energy Sources;

Appropriate Technology; Biomass Conversion; Energy

Conversion; Energy Storage; Heat and Energy Transfer

Material Fesearch; Photovoltalcs

General Physics

Instructor:

Vetler, Willard

(607) 746.4374

Course Number:

9521

Physical Sciences

Program or Curriculum: Construction Tech./Civil Tech.

Credits: 3

Freshman or Sophomore

Student Level:

15 Weeks, 4.0 hrs per week

Contact Hours:

60

Classroom: $\quad 30$

Laboratory: $\quad 30$

Topics Covered Extensively: Alternate Energy Sources;

Appropriate Technology; Energy Conservation; Energy

Conversion; Energy Storage; Heat and Energy Transfer; Intro. to Solar Energy

Average Enrollment:

100

General Physics 9522

Instructor:

Course Number:

Department:

\section{Vetter, Willard}

(607) $746-4374$

952 ?

Physical Sciences

Program or Curriculum: Construction Tech./Civil Tech.

Credits: 3

Student Level:

Duration:

Contact Hours:

Freshman or Sophomore

15 Weeks, $4.0 \mathrm{hrs}$ per week

60

30

30

Laboratory:

Topics Covered Extensively; Alternate Energy Sources;

Appropriate Technology; Energy Conservation; Energy

Conversion; Energy Storage; Heat and Energy Transfer;
Intro. to Solar Energy

Average Enrollment: 100

Mechanical Equipment for Buildings

Hampel, John

Course Number: $\quad$ (607) $746-4386$

Department: Construction Techuolugy

Program or Curriculum: Construction Tech./Civil Tech.

Credits:

Student Level:

Duration:

Contact Hours:

Classroom:

Laboratory:

4

Freshman or Sophomore

15 Weeks, 4.0 hrs per week

60

45

30

Topics Covered Extensively: Energy Conservation; Energy

Conversion; Energy Storage; Heat and Energy Transfer;

Plumbing Techniques

Number of Times Taught: 20

Average Enrollment: $\quad 65$

Thermodynamics and Heating

Instructor:

Hampel, John

(607) $746-4386$

Course Number: $\quad 3711$

Department: Construction Technology

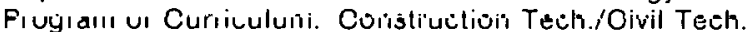

Credits:

Freshman or Sophomore

Duration:

Contact Hours:

Classroom:

15 Weeks, $4.0 \mathrm{hrs}$ per week

Topics Covered Extensively: Energy Conservation; Energy

Conversion; Energy Storage; Heat and Energy Transfer;

Plumbing Techniques

Number of Times Taught: 20

Average Enrollment: $\quad 65$

Water Resources

Instructor:

Singer, Darrell

Course Number: $\quad$ (607) $746-4391$

Department: Civil Technology

Program or Curriculum: Construction Tech./Civil Tech

Credits:

Slunderit I revel:

Duration:

Contact Hours:

Classroom:

Laboratory:
Topics Covered Extensively: Alternate Energy Sources;

Biomass Conversion; Energy Conservation; Energy Cun-

version; Energy Storage

Number of Times Taught: 20

Average Enrollment: $\quad 30$

\section{NORTH CAROLINA}

Cape Fear Technical Institute [5320]

Wilmington, North Carolina 28401

(919) 343-0481

Programs and Curricula

General Occupational Technologies

Degree: AD, General Occupational

Technologies

Contact: $\quad$ Stiles, W. O./ Averette, R

(919) 343-0481

Solar Related Courses

Introduction to Energy Resources

Instructor: Bordeaux, Ralph

(919) 343-048

Department: Engineering Occupational

Program or Curriculum: General Occupational

Credits: 3

Student Level: $\quad$ All levels 


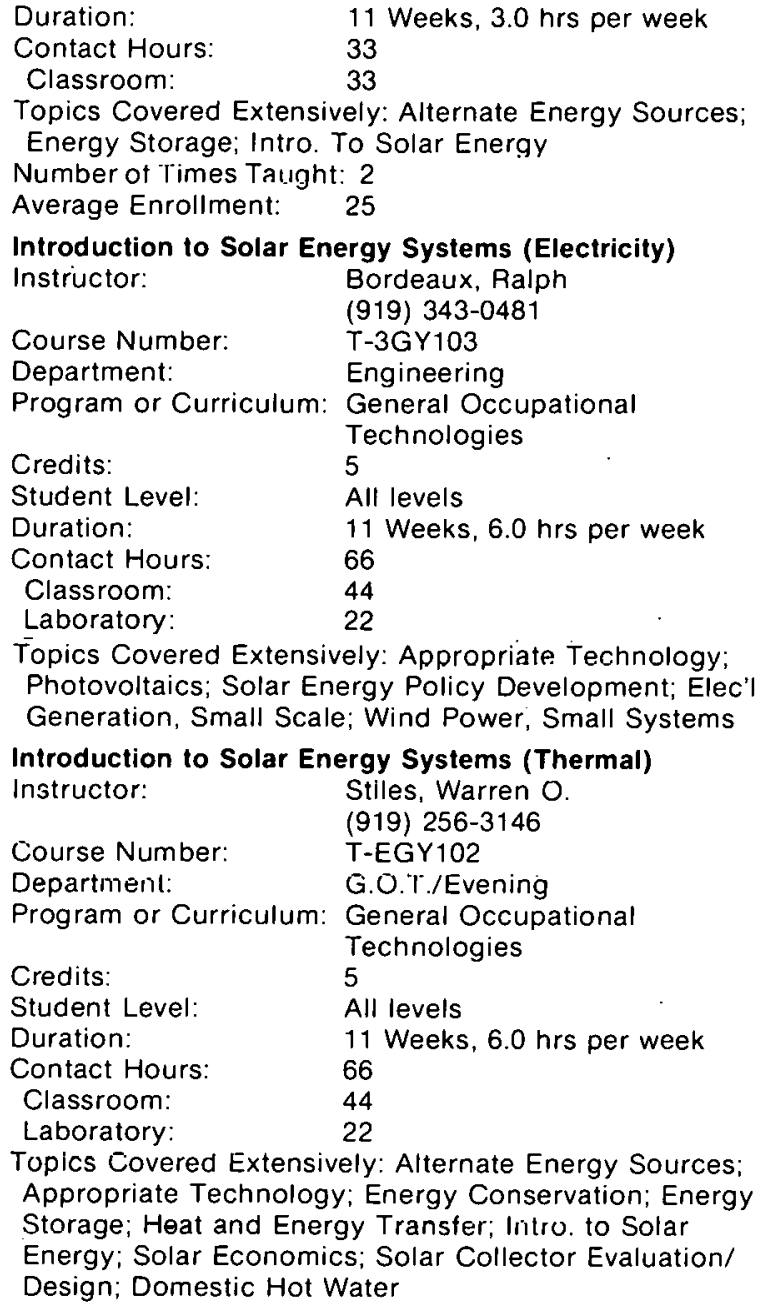

\section{Southwestern Technical Institute [8466] Sylva, North Carolina 28779 \\ (704) 586-4091}

Programs and Curricula

Solar Energy Systems-Residential and

Commercial Construction

$\begin{array}{ll}\text { Degree: } & \text { Certificate of Completion } \\ \text { Contact: } & \text { Liming. Glenn }\end{array}$ (7ח4) 586-4ח9.1

Students Taking or Completing Offering:

lisilaller-Residential (Solar System), Installer-Commercial (Solar System), Solar Technician

\section{Solar Related Courses}

Introduction to Solar Concepts

$\begin{array}{ll}\text { Instructor: } & \begin{array}{l}\text { Liming, Glenn } \\ \text { (704) 586-4091 }\end{array} \\ \text { Course Number: } & \text { CAR 1120 } \\ \text { Department: } & \text { Industrlal/Vocational } \\ \text { Program or Curriculum: Solar Energy Systems-Res. and } & \begin{array}{l}\text { Comm. Construction } \\ \end{array} \\ \text { Ciedils: } & 4 \\ \text { Student Level: } & \text { Freshman or Sophomore } \\ \text { Duration: } & 11 \text { Weeks, 6.0 hrs per week } \\ \text { Contact Hours: } & 66 \\ \text { Classronm: } & 33 \\ \text { Laboratory: } & 33 \\ \text { Number of Times Taught: } 1 \\ \text { Average Enrollment: } & 8 \\ \text { Sular Collecior } & \\ \text { Instructor: } & \text { Liming, Glenn } \\ & (704) 586-4091\end{array}$

Course Number:

Department:

Program or Curriculum: Solar Energy Systems-Res. and

Credits:

Sludent Level:

Duration:

Contact Hours:

Classroom:

Laboratory:

Topics Covered Extensively: Solar Collector Evaluation

Design

Number of Times Taught: 1

Average Enrollment: 8

Solar Energy Heating Systems

Instructor: Liming, Glenn

Course Number: $\quad$ CAR 1122

Department: Industrial/Vocational

Program or Curriculum: Solar Energy Systems-Res. and

Credits:

Student Level: $\quad$ Freshman or Sophomore

Duration: . 11 Weeks, $6.0 \mathrm{hrs}$ per week

Cuntact Hours: $\quad 66$

Classroom: $\quad 33$

Laboratory: $\quad 33$

Topics Covered Extensively: Intro. to Solar Energy;

Swimming Pool Heating

Number of Times Taught: 1

Average Enrollment: 8

\section{NORTH DAKOTA}

Bismarck Junior College [2988]

Bismarck, North Dakota 58501

(701) 223-4500

\section{Programs and Curricula}

\section{Solar Heating}

Degree:

Contact:

Certificate of Completion McKinney, David

(701) 255-0566

Students Taking or Completing Offering:

Plumber, Sheet Metal Worker

\section{Solar Related Courses}

Solar Energy

Instructor:

McKinney, David

(701) 255-0566

Department: Heating, Refrigeration, and Air Conditioning

Program or Curriculum: Solar Heating

Credits:

Student Level:

Duration:

Contaret Hours:

Classroom:

Laboratory:

8

Freshman or Sophomore

8 Weeks, 32.0 his per week 256

63

193

Topics Covered Extensively; Plumbing Techniques; Sheet Metal Techniques; Solar Home Construction; Solar Systems Design; Solar Systems Installation; Solar Systems Maintenance; Solar Systems Testing and Evaluation; Domestic Hot Water; Space Healing; Space Cooling

North Dakota State School of Science [2996] Wahpeton, North Dakota 58075

(701) $671-1130$

\section{Programs and Curricula}

Envlronmental Systems Design

Degree:

Certificate, Diploma

Contact:

Whitcomb Larry

(701) $671-2529$

Students Taking or Completing Offering:

Installer-Residential (Solar System), Installer-Commercial (Solar Systems), Trade Specialty, Plumber, Sheet Metal Worker 
Solar Related Courses

Systems and Equipment

Whitcomb, Larry

(701) $671-2529$

Course Number

ESD 203

Department: Environmental Systems

Credits:

Gtudent Level:

All levals

Duration:

Contact Hours:

12 Weeks, 5.0 hrs per week

60

Classroom:

Topics Covered Extensively: Alternate Energy Sources; Heat and Energy Transfer; Intro. To Solar Energy; Plumbing Techniques; Sheet Metal Techniques; Solar Home Construction; Solar Collector Evaluation/Design; Solar Systems Installation; Solar Systems Maintenance Number of Times Taught: 1

Average Enrollment: 25

\section{OHIO}

\section{North American Heating and Air Conditioning Wholesalers Association \\ Homc Study Inetitute 1661 West Henderson Columbus. Ohio 43220 \\ Programs and Curricula \\ *Home Study Program \\ Contact: Healy, James (614) 459-2100 \\ Students Taking or Completing Offering: Solar Technician}

\section{OKLAHOMA}

None

\section{OREGON}

Linn-Benton Community College [6938] Albany, Oregon 97321

(50'3) 928-2361

Programs and Curricula

Engineering Technology - Solar Energy Option

Degree: $\quad A D$, Engineering Tcch

Contact: Miller, Dave

(503) $928-2361$

\section{Solar Related Courses}

Alternate Energy Sources

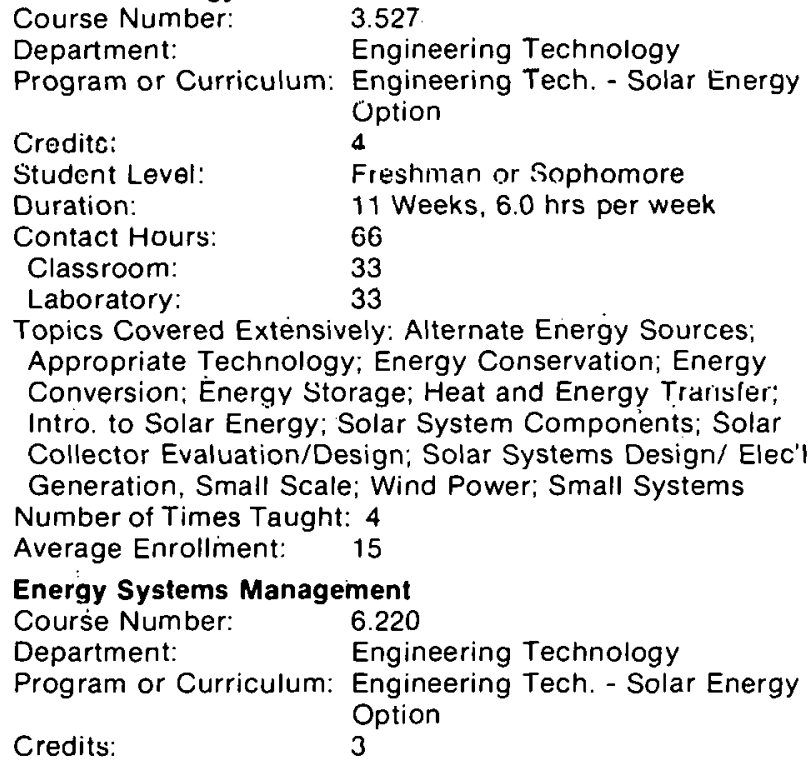

$\begin{array}{ll}\text { Student Level: } & \text { Freshman or Sophomore } \\ \text { Duration: } & 11 \text { Weeks, } 3.0 \text { hrs per week } \\ \text { Contact Hours: } & 33 \\ \text { Classiouin: } & 33 \\ \text { Topics Covered Extensively: Energy Conservation; Energy } \\ \text { Conversion: Heat and Energy Transfer; Intro. to Solar } \\ \text { Systems Maintenance; Solar Systems Testing and Evalua- } \\ \text { tion; Elec'l Generation, Central; Elec'l Generation, Small } \\ \text { Scale; Plucess Hedl, lidusliabl; Goacc I lcating; Space } \\ \text { Cooling; Wind Power, Central Systems; Wind Power, } \\ \text { Small Systems }\end{array}$

Solar Energy

$\begin{array}{ll}\text { Course Number: } & 6.221 \\ \text { Department: } & \text { Engineering Technology }\end{array}$

Program or Curriculum: Engineering Tech. - Solar Energy

Credits Options

Student Level:

Duration:

Contact Hours:

Classroom:

Freshman or Sophomore

11 Weeks, $3.0 \mathrm{hrs}$ per week

Topics Covered Extensively: Biomass Conversion: Energy Conservation; Energy Conversion; Energy Storage; Heat and Energy Transfer; Intro. to Solar Energy; Materials Rocoarsh; Passive Solar Terhnnlngy: Snlar System

Components; Solar Economics; Solar Home Construction; Solar Collector Evaluation/Design; Solar Systems Design; Sular Systems Testing and Evaluation; Domestic Hot Water Elec'l Generation, Small Scale; Spacc Heating; Wind Power, Small Systems

Number of Times Taught: 2

Average Enrollment: $\quad 12$

\section{PENNSYLVANIA}

Keystone Junior College [3280]

La Plume Pennisylvania 18440

(707) 945-5141

Programs and Curricula

Solar Engineering Technology

Degree:

AU, Applied Sclenlie liı Bula

Contact: Fngineering Technology Kutch, Dennis/Cupilleri, Tom (717) $945-5141$

Students Taking or Completing Offering

Solar Technician

\section{Solar Related Courses}

Sizing, Installation \& Operation - Solar Heating

(Residential Buildings)

Instructor:

Kutch, Dennis

(717) $945-5141$

Department: Solar Energy Sludy \& Res. Cnt.

Program or Curriculum: Solar Engineeririy Technology

Student Level:

All levels

Duration:

Contact Hours: 2 Weeks, $36.0 \mathrm{hrs}$ per week

Classroom:

Laboratory: 72

Topics Covered Extensively: Energy Conservation; Plumbing Techniques; Sheet Metal Techniques; Solar System Components; Solar Home Construction; Solar Systems Installation; Solar Systems Maintenance; Solar Systems Testing and Evaluation: Domestic Hot Water: Swimming Pool Heating: Space Heating

Solar Hydronic Systems/Solar Air Systems

Instructor: Kutch, Dennis

(717) $945-5141$

Department: Solar Energy Study \& Res. Cnt.

Program or Curriculum: Solar Engineering Technology

Credits:

Student Level:

Duration:

Contact Hours:
All levels

16 Weeks, $3.0 \mathrm{hrs}$ per week 
Classroom:

30

Laboratory: 18

Topics Covered Extensively: Energy Conservation; Heat and Energy Transfer: Plumbing Techniques; Sheet Metal Techniques; Solar System Components; Solar Economics: Solar Systems Installation; Solar Systems Maintenancé; Solar Systems Testing and Evaluation; Domestic Hot Water; Swimming Pool Heating; Space Heating

Training - Design of Solar Heating Systems for Buildings Instructor: Kutch, Dennis (717) $945-5141$

Department: Solar Energy Study \& Res. Cnt.

Program or Curriculum: Solar Engineering Technology

Student Level: Junior or Senior

Duration: 2 Weeks, 36.0 hrs per week

Contact Hours: 72

42

Classroom: 30

Laboratory:

Topics Covered Extensively: Energy Conservation; Energy Storage; Heat and Energy Transfer; Solar Economics; Solar Home Construction; Solar Collector Evaluation/Design; Solar Systems Design; Domestic Hot Water; Space Heating

\section{Pennsylvania Institute of Technology [90180] 414 Sansom St. Upper Darby, Pennsylvania 19082}

\section{Programs and Curricula}

\section{Energy Technology}

Degree:

Contact:

AD, Specialized Technology

Students Taking or Completing Offering:

Solar Technician, Electrician

\section{Solar Related Courses}

Advanced Solar Design

Iristructor:

Thomas, Richard

(215) 352-7100

Course Number: $\mathrm{K}$

Program or Curriculum: Energy Technology

Credits:

2

Student Level:
Duration:

Contact Hours:

High School Graduate

12 Weeks, 4.0 hrs per week 48

Topics Covered Extensively: Solar Coliector Evaluation/ Design; Solar Systems Design

Number of Times Taught: 1

Average Enrollment: $\quad 35$

\section{Basic Solar Design}

Instructor:

Thomas, Richard $\mathrm{B}$. (215) $352-7100$

Course Number:

Program or Curriculum: Energy Technology Credits:

Student Level:

Duration:

Contact Hours:

High School Graduate

12 Weeks, 5.0 hrs per week 60

Topics Covered Extensively: Energy Storage; Heat and

Energy Transfer; Intro. to Solar Energy; Passive Solar

Technology; Solar Home Construction; Solar Collector Evaluation/Design

Number of Times Taught: 1

Average Enrollment: $\quad 35$

\section{Energy Conversion}

Instructor:

Thomas, Richard (215) $352-7100$

Course Number: B

Program or Curriculum: Energy Technology

Credits:

Student Level:

4

Duration:

Contact Hours:

High School Graduate

12 Weeks, $5.0 \mathrm{hrs}$ per week 60

Topics Covered Extensively: Alternate Energy Sources:

Energy Conversion; Intro. to Solar Eneray; Wind Power.

Sma!! Systoms

Number of Times Taught: 1

Average Enrollment: $\quad 35$
Pennsylvania State University - Shenango
Valley Campus

Sharon, Pennsylvania 16146

(412) $981-1640$

Programs and Curricula

Solar Heating and Cooling Technology

Degree:

Short Course Certificate

Contact:

(412) $981-1640$

Solar Related Courses

Fundamentals of Solar Energy

Instructor: Houlinan, J. F.

(421) $981-1640$

Department: Physics

Program or Curriculum: Solar Heating and Cooling Tech.

Credits:

Student Level: $\quad$ Freshman or Sophomore

Duration:

Contact Hours: 10 Weeks, 2.0 hrs. per week

Classroom:

Laboratory:

15

Topics Covered Extensively: Energy Conversion; Energy Storage; Heat and Energy Transfer; Intro. to Solar Energy: Passive Solar Technology; Photovoltaics; Solar System Components; Solar Home Construction; Solar Collector Evaluation/Design; Solar Systems Design; Space Heating; Space Cooling: Wind Power, Central Systems

Number of Times Taught: 2

Average Enrollment: 12

Introduction to Solar Energy

Instructor:

Houlihan, J.F. (412) $981-1640$

Course Number: $\quad$ PHYS. 297

Department: Physics

Program or Curriculum: Solar Heating and Cooling Tech.

Credits:

Duration:

Contact Hours:

Classroom: 28

Laboratory

Freshman or Sophomore 10 Weeks, 3.0 hrs per week

28

Topics Covered Extensively: Intro. to Solar Energy; Space Heating; Wind Power, Central Systems

Number of Times Taught: 2

Average Enrollment: 15

Solar Heating and Cooling Technology

Instructor: Houlihan, J. F.

(412) $981-1640$

Course Number: $\quad$ PHYS. 297

Department: Physics

Prugram or Curriculum: Solar Heating and Cooling Tech.

Credits: 1

Student Level: $\quad$ All levels

Duration:

Classroom:

2 Weeks, $40.0 \mathrm{hrs}$ per week

Laboratory:

60

Topics Covered Extensively: Energy Conservation; Energy Storage; Heat and Energy Transfer; Intro. to Solar Energy; Marketing/Market Analysis; Passive Solar Technology; Solar System Components; Solar Home Construction; Solar Collector Evaluation/Design; Solar Systems Design; Solar Systems Installation; Solar Systems Maintenance: Domestic Hot Water; Space Heating, Space Cooling Number of Times Taught: 1

Average Enrollment: $\quad 30$ 
Installation; Solar Systems Maintenance; Solar Systems Testing and Evaluation; Domestic Hot Water; Swimming Pool Heating; Space Heating

Number of Times Taught: 2

Average Enrollment: 25

Triangle Institute of Technology, Inc. [90110] 635 Smithfield St.

Pittsburgh, Pennsylvania 15222

(412) $255-6170$

Programs and Curricula

Solar Energy Systems

Degree:

Contact:

AD, Specialized Technology

Knoyer, Ralph

(412) 255-6170

Students Taking or Completing Offering: Solar Technician; Electrician, Plumber, Sheet Metal Worker

\section{Solar Related Courses}

Solar Energy Systems

Instructor:

Knoyer, Ralph

(412) $255-6170$

Course Number:

400.0

Department:

Refrig., Heat., Vent., and Air Cond.

Program or Curriculum: Solar Energy Systems

Credits:

Student Level:

Duration:

Contact Hours:

10

High School Graduate

16 Weeks, 25.0 hrs per week

Laboratory: $\quad 300$

Topics Covered Extensively: Solar Systems Design: Sular

Systems Installation; Solar Systems Maintenance; Solar

Systems Testing and Evaluation

\section{RHODE ISLAND}

None

\section{SOUTH CAROLINA}

\section{Beaufort Technical Education Center [9910]}

Bequfort, South Carolina 28902

(803) 524-3380

Programs and Curricula

Refrigeration and Air Conditioning - Solar Energy

Applications

Degree:

$A D$, Refrigeration and Air Conditioning, General Technology

Contact: Spivey, Edward $F$ (803) 524-0148

Students Taking or Completing Offering: Installer-

Residential (Solar System), Solar Technician, Trade Specialty

\section{Solar Related Courses}

Solar Energy Application

Instructor:

Spivey, E.F

(803) 524-0148

Course Number: $\quad$ ARC 240

Department: Refrigeration and Air Conditioning

Program or Curriculum: Refrigeration and Air ConditioningSolar Energy Appli.

Credits:

Student Level

Duration:

High School Graduate

Contact Hours: $\quad 66$

Classroom: $\quad 33$

Laboratory: $\quad 33$

Topics Covered Extensively: Heat and Energy Transfer
Florence Darlington Tech [3990]. Florence, South Carolina 29502 (803) $662-8151$

Programs and Curricula

Conversion of Solar Energy

Degree: Climate Control

Contact: Jackson, Edward (803) 662-8151

\section{Solar Related Courses}

Conversion of Solar Energy

Instructor: Jackson, Edward

(803) 662-815

Department: Industrial Trades - Climate Control

Program or Curriculum: Conversion of Solar Energy

Credits

Student Level: High School Graduate

Duration: $\quad 11$ Weeks, $6.0 \mathrm{hrs}$ per week

Contact Hours: $\quad 66$

Classroom: $\quad 33$

Laboratory: $\quad 33$

Topics Covered Extensively: Alternate Energy Sources;

Appropriate Technology; Intro. to Solar Energy; Solar

Systems rommnnents: Solar Collector Evaluation/Design;

Solar Systcms Design; Solar Systems Installation; Solar

Systems Maintenance: Domestic Hot Water

Number of Times Taught: 4

Average Enrollment: 25

Trident Technical College [8818] PO Box 10367

Charleston, South Carolina 29411

(803) 553-2375

\section{Programs and Curricula}

Air Conditioning - Refrigeration

$\begin{array}{ll}\text { Degree: } & \text { Air Conditioning - Refrigeration } \\ \text { Contact: } & \text { Moore, James L. . }\end{array}$

(803) 572-6180

Students Taking or Completing Offering: Installer-

Residential (Solar System)

Solar Related Courses

Solar Heating

Instructor:

Moore, James $1 .$.

Department: $\quad$ (803) $572-6180$

Frogram or Curriculum: Ail Conditioning -... Refrigeration

Student Level: High School Graduate

Duration:

Contact Hours:

3 Weeks, $30.0 \mathrm{hrs}$ per week

Classroom:

90

Lahoratory:

Topics Covered Extensively: Heat and Energy Transfer; Solar System Components; Solar Collector Evaluation/

Design; Domcstic Hot Wator; Spaco Heating

York Technical College [3996]

Rock Hill, South Carolina 29730

(803) 328-3843

Programs and Curricula

Conversion of Solar Energy

Degree: Air Conditioning, Refrigeration,

Contact:

and Heating

White, Lacy

(803) 324-3130

Students Taking or Completing Offering: Installer-

Residential (Solar System), Installer-Commercial (Solar

System), Trade Specialty

Solar Related Courses

Conversion of Solar Energy

Instructor:

White, Lacy. 
Topics Covered Extensively: Plumbing Techniques; Solar Systems Installation: Domestic Hot Water; Space Heating Number of Times Taught: 1

Average Enrollment: 18

\section{SOUTH DAKOTA}

None

\section{TENNESSEE}

\section{Motlow State Community College [6836]}

Tullahoma, Tennessee 37388

(615) 455-8511

\section{Programs and Curricula}

Energy Engineering Technology

Degree: $\quad$ AD, Engineering Technology -

$\begin{array}{ll}\text { Energy Engineering } \\ \text { Contact: } & \text { Thornton, Otis B. }\end{array}$

(615) $455-8511$

Students Taking or Completing Offering: Installer-

Residential (Solar System), Researcher, Solar Technician

Solar Related Courses

Solar Energy Applications

Instructor

Lowndes, Richard

Course Number:

(615) 455-8511

Department:

ERG 205

Program or Curriculum: Energy Engineering

Credits:

Student Level: All levels

Duration: $\quad 10$ Weeks, $5.0 \mathrm{hrs}$ per week

Contact Hours: $\quad 50$

Classroom: $\quad 20$

Laboratory:

30

Topics Covered Extensively: Energy Storage; Heat and Energy Transfer; Intro. to Solar Energy; Solar System Components; Solar Economics; Solar Home Construction; Solar Collector Evaluation/Design; Solar Systems Design; Solar Systems Installation; Solar Systems Testing and Evaluation; Domestic Hot Water; Space Heating

\section{Solar Energy Theory}

Instructor:

Lowndes, Richard

(615) 455-8511

Course Number:

ERG 204

Department:

Career Education

Program or Curriculum: Energy Engineering Technology

Credits:

Student Level:

Duration:

Contact Hours:

All levels

10 Weeks, $5.0 \mathrm{hrs}$ per week

Classroom:

Laboratory:

50

20

30

Topics Covered Extensively: Energy Storage; Heat and Energy Transfer; Intro. to Solar Energy; Solar Systems Components; Solar Economics; Solar Home Construction: Solar Collector Evaluation/Design; Solar Systems Design; Solar Systems Installation; Solar Systems Testing and Evaluation; Domestic Hot Water; Space Heating

\section{TEXAS}

\section{Central Texas College [4003]}

Killeen, Texas 76541

(817) 526-1211

\section{Programs and Curricula}

\section{Solar Energy Systems Specialist}

Degree:

Certificate of Completion

Contact:

Tresler, Clarence

(817) 526-1236

Students Taking or Completing Offering: InstallerResidential (Solar System), Installer-Commercial (Solar Syctem), Eolar Technician, Trade Sptiially

\section{Solar Energy Systems Technology} Degree: AD, Applied Sciencé
Contact: Tesler, Clarence

(817) 526-1236

Students Taking or Completing Offering: Installer-

Residential (Solar System), Installer-Commercial (Solar

System), Solar Technician, Trade Specialty

\section{Solar Related Courses}

Principles of Solar Energy

Instructor:

Tresler, Clarence

Course Number

(817) $526-1236$

Department:

SESY 1314

Industrial Technology

Program or Curriculum: Solar Energy Systems Technology/

Credits: Solar Energy Systems Specialist

Student Level:

Duration:

Contact Hours:

Freshman or Sophomore 16 Weeks, 3.0 hrs per week

Classroom: 48

48

Topics Covered Extensively: Intro. to Solar Energy Number of Times Taught: 2

Average Enrollment: 25

Solar Cooling Systems Instructor:

\section{Tresler, Clarence} (817) $526-1236$

Course Number SESY 241

Department: Industrial Technology

Program or Curriculurr: Solar Energy Systems Technology Credits:

Student Level: $\quad$ Freshman or Sophomore

Duration:

Contact Hours:

Classroom:

16 Weeks, 6.0 hrs pcr week

Laboratory: 96

48

Topics Covered Extensively: Energy Storage; Heat and Energy Transter; Plumbing Techniques; Solar System Components; Solar Home Construction; Solar Collector Evaluation/Design; Solar Systems Design; Solar Systems Installation; Solar Systems Maintenance; Solar Systems Testing and Evaluation

\section{Solar Energy Special Projects}

Instructor:

Tresler, Clarence

(817) $523-1236$

Course Number:

SESY 231

Department: Industrial Technology

Program or Curriculum: Solar Energy Systems Technology Credits:

Student Level: 3

Duration

Contas. Hours:

Classroom:

Laboratory:

Freshman or Sophomore 16 Weeks, $6.0 \mathrm{hrs}$ per week 96

16 80

Topics Covered Extensively: Energy Conversion; Energy Storage; Heat and Energy Transfer; Plumbing Techniques; Solar System Components; Solar Home Construction; Solar Collector Evaluation/Design; Solar Systems Design; Solar Systems Installation; Solar Systems Maintenance; Solar Systems Testing and Evaluation; Domestic Hot Water: Swimming Pool Heating; Space Heating: Space Cooling

Solar Heating Systems

Instructor:

Tresler, Clarence

Course Number (817) $52.6-1236$

Department:

Program or Curriculum: Solar Energy Systems Technologyi

Credits: Solar Energy Systems Specialist

Student Level:

Duration:

Contact Hours:

Classroom:

Laboratory:

Freshman or Sophomore 16 Weeks, $6.0 \mathrm{hrs}$ per week

Topics Covered Extensively: Energy Storage; Heat and Energy Transfer; Plumbing Techniques; Solar System Components; Solar Home Construction; Solar Collector Evaluation/Design; Solar Systems Design; Solar Systems 
Navarro College [3593]
Corsicana, Texas 75110

(214) 874-6501

Programs and Curricula

Solar Energy Installers-Mechanics

Degree:

OTHER, Solar Energy Installers, Mechanics

Contact

kidsplzyk, Eıluesl

(214) 874-6501

Students Taking or Completing Offering: Installer-

Residential (Solar System), Installer-Commercial (Solar

System)

Solar Engineering Technology

Degree:

AD, Applied Science - Solar

Engineering Technology

Contact:

Myers, Arthur

(214) 874-6501

Students Taking or Completing Offering: Solar Technician

\section{Solar Related Courses}

\section{Collector and Energy Storage}

Instructor:

Myers, Arthur

(214) 874-6501

Proaram or Curriculum: Solar Engineering Technology

Crcodits:

4

Student Level:

Freshman or sopnomore:

16 Weeks, $6.0 \mathrm{hrs}$ per week 96

Contact Hours:

Classroom:

Laboratory:

64

Topics Covered Extensively: Energy Conservation; Energy

Conversion; Energy Storage; Heat and Energy Transfer

Materials Research; Passive Solar Technology; Plumbing

Techniques; Sheet Metal Techniques; Solar System

Components; Solar Home Construction; Solar Collector

Evaluation/Design; Solar Collector Evaluation/Design;

Solar Systems Design; Solar Systems Installation; Solar

Systems Maintenance; Domestic Hot Water; Swimming

Pool Heating; Space Heating; Space Cooling

Collectors, Energy Storage, Installation and Service

Instructor: Norman, Albion

(214) 874-6501

Courco Number: $\quad$ SE1DA4

Program or Curriculum: Solar Energy Installers/Mechanics

Credits:

Student Level:

Duration:

Contact Hours:

4

Freshman or Sophomore

16 Weeks, $6.0 \mathrm{hrs}$ per week

96

Classroom:

l.aboratory:

64

Topics Covered Extensively: Alternate Energy Sources; Appropriate Technology; Energy Conservation; Energy Storage: Heat and Energy Transter; Intro. to Solar Energy Passive Solar Technology; Plumbing Techniques; Sheet Metal Techniques; Solar System Components; Solar Collector Evaluation/Design; Solar Systems Design; Solar

Systems Installation; Solar Systems Maintenance; Solar

Systems Testing and Evaluation; Domestic Hot Water

Space Heating; Space Cooling

Economics, Codes, Legal, Consumerism

Instructor: Myers, Arthur

(214) 874-6501

Department: Solar Energy

Program or Curriculum: Solar Engineering Technology

Credits:

Student Level:

Duration:

Contact Hours:

Freshman or Sophomore

16 Weeks, $2.0 \mathrm{hrs}$ per week

32

Classroom:

Topics Covered Extensively: Alternate Enérgy Sources:

Appropriate Technology; Marketing/Market Analysis;

Solar Economics; Solar Home Construction; Solar Law/

Legislation; Solar Collector Evaluation/Design; Solar

Systems Testing and Evaluation; Domestic Hot Water;

Swimming Pool Heating; Space Heating; Space Cooling
Energy Science 1

Instructor:

Department:

Myers, Arthur

(214) 874-6501

Solar Energy

Program or Curriculum: Solar Engineering Technology

Credits:

Student Level: $\quad$ Freshman or Sophomore

Duration:

rontart Hnurs:

16 Weeks, $6.0 \mathrm{hrs}$ per week

Classroom:

Laboratory:

48

48

Topics Covered Extensively: Alternate Energy Sources; Appropriate Technology; Energy Conservation; Energy Conversion; Energy Storage; Heat and Energy Transfer; Intro. to Solar Energy; Plumbing Techniques; Sheet Metal Techniques; Solar System Components; Solar Systems Design; Solar Systems Installation; Solar Systems Maintenance; Domestic Hot Water: Space Heating; Space

Cooling

Energy Science II

Instructor:

Myers, Arthur

(214) 874-6501

Department: Solar Energy

Program or Curriculum: Solar Engineering Technology

Credits:

Student Level

Uuration:

Contact Hours:

Freshmán or sophomore

$16 \overline{\text { Weeks, }} 0.0 \mathrm{lils}$ per week

Classroom:

Laboratory: 96

48

48

Topics Covered Extensively: Alternate Energy Sources; Appropriate Technology; Energy Conservation; Energy Conversion; Energy Storage; Heat and Energy Transfer; Intro. to Solar Energy; Plumbing Techniques; Sheet Metal Techniques; Solar System Components; Solar Systems Design; Solar Systems installation; Solar Systems Maintenance; Domestic Hot Water; Space Heating; Space Cooling

Introduction to Solar Heating and Cooling

Instructor: Norman, Albian

(214) 874-6501

Course Number: SE1013

Department: Occupational Education

Program or Courriculum: Solar Energy Installers/Mechanics

Credits:

Student Level:

Duration:

Contact Hours: 3 Freshman or Sophomore 16 Weeks, $3.0 \mathrm{hrs}$ per week 48

Classroom:

Topics Covered Extensively: Alternate Energy Sources; Appropriate Technology; Energy Conservation; Heat and Energy Transfer; Intro. to Solar Energy; Passive Solar Technology; Plumbing Techniques; Solar System Components;-Solar Home Construction; Sular Systems

Design; Domestic Hot Water; Elec'l Generation, Small

Scale; Space Heating; Space Cooling

Number of Times laught: 1

Average Enrollment: 23

Materials and Fabrication

Instructor:

Vaughn, Ralph

(214) 874-6501

Course Number: SE1034

Department: Occupational Education

Program or Curriculum: Solar Energy Installers/Mechanics

Credits:

Student Level: $\quad$ Freshman or Sophomore

Duration: $\quad 16$ Weeks, $6.0 \mathrm{hrs}$ per week

Contact Hours: $\quad 96$

Classroom: 32

Laboratory: $\quad 64$

Topics Covered Extensively: Energy Storage; Heat and Energy Transfer; Materials Research; Plumbing Techniques; Sheet Metal Techniques; Solar System Components; Solar Home Construction; Solar Systems Design Solar Systems Installation; Solar Systems Maintenance; Solar Systems Testing and Evaluation; Domestic Hot Water; Space Heating; Spacc Cooling 
Materials and Material Handling

Myers, Arthur

(214) 874-6501

Program or Curriculum: Solar Engineering Technology Credits:

3

Student Level: $\quad$ Freshman or Sophomore

Duration:

Contact Hours:

16 Weeks, 5.0 hrs per week

80

16

Classroom:

Laboratory

Topics Covered Extensively: Materials Research; Plumbing

Techniques; Sheet Metal Techniques; Domestic Hot

Water; Space Heating; Space Cooling

\begin{tabular}{|c|c|}
\hline $\begin{array}{l}\text { Non-residential Applicati } \\
\text { Instructor: }\end{array}$ & $\begin{array}{l}\text { Ions \& Future Te } \\
\text { Myers, Arthur } \\
\text { (214) 874-6501 }\end{array}$ \\
\hline$n t:$ & Solar Energy \\
\hline Curriculum: & Solar Engineering Technology \\
\hline & \\
\hline Level: & Freshman or Sophomore \\
\hline & \\
\hline urs: & 8 \\
\hline & \\
\hline & \\
\hline \multicolumn{2}{|c|}{$\begin{array}{l}\text { Topics Covered Extensively: Altermate Energy Sources; } \\
\text { Appropriate Technology; Passive Solar Technology; } \\
\text { Photovoltaics; Solar System Components; Solar Systems } \\
\text { Design; Solar Systems Installation; Solar Systems Mainte- } \\
\text { nance; Swimming Pool Heating; Elec'l Generation, Cen- } \\
\text { tral; Elec'l Generation, Small Scale; Process Heat, Agricul- } \\
\text { tural; Process Heat, Industrial; Space Heating; Space } \\
\text { Cooling }\end{array}$} \\
\hline
\end{tabular}

\section{Operational Diagnosis}

Instructor:

Myers, Arthur

(214) 874-6501

Solar Energy

Department:

Solar Enginéering Technology

Program or Curriculum:

Credits:

Student Level

Freshman or sophomore

16 Weeks, 5.0 hrs per week

Duration:

Contact Hours:

32

Classroom:

Topics Covered Extensively: Plumbing Techniques; Sheet

Metal Techniques; Solar System Components; Solar

Collector Evaluation/Design; Solar Systems Design; Solar

Systems Installation; Solar Systems Maintenance; Solar

Systems Testing and Evaluation

Sizing Design and Retrofit

Myers, Ailliur

(214) 874-6501

Department:

Solar Energy

Program or Curriculum: Solar Engineering Technology

Credits:

Student Level

Duration:

Currlact Hours:

Freshman or Sophomore

16 Weeks, 6.0 hrs per week

Classroom:

96

48

Laboratory

48

Topics Covered Extensively: Solar Home Construction

Solar Systems Design; Solar Systems Installation; Solar

Systems Testing and Evaluation; Domestic Hot Water;

Space Heating; Space Cooling

Solar Heating and Cooling Systems

Instructor:

Myers, Arthur

214) 874-650

Course Number:

SE1064

Department:

Occupational Education

Program or Curriculum: Solar Energy Installers/Mechanics

Credits:

Student Level:

Duration:

Contact Hours:

Classroom:

Laboratury:
Freshman or Sophomore

16 Weeks, 6.0 hrs per week

96

32

64
Topics Covered Extensively: Alternate Energy Sources: Appropriate Technology; Energy Conservation; Energy Storage; Heat and Energy Transfer; Intro. to Solar Energy; Materials Research; Passive Solar Technology; Plumbing Techniques; Sheet Metal Techniques; Solar System Components; Solar Collector Evaluation/Design; Solar Systems Installation; Solar Systems Maintenance; Solar Systems Testing and Evaluation; Domestic Hot Water; Process Heat, Agricultural; Process Heat, Industrial; Space Heating; Space Cooling

Average Enrollment: 15

Solar Practicum

Instructor: Myers, Arthur

(214) 874-6501

Department: Solar Energy

Program or Curriculum: Solar Engineering Technology

Credits:

Student Level:

Duration:

Contact Hours:

Laboratory:

Freshman or Sophomore

16 Weeks, 3.0 hrs per week

48

48

Topics Covered Extensively: Alternate Energy Sources:

Appropriate Technology; Intro. to Solar Energy; Materials

Research; Plumbing Techniques; Sheet Metal Techniques;

Solar Systems Design; Solar Systems Installation; Solar

Systems Maintenance; Solar Systems Testing and Evalua-

tion; Domestic Hot Water; Space Heating; Space Cooling

Technical Surveys of Energy Sources

Instructor: Myers, Arthur

(214) 874-6501

Department: Solar Energy

Program or Curriculum: Solar Engineering Technology

Credits:

3

Student Level:

Duration:

Contact Hours:

Freshman or Sophomore

Classroom: 16 Weeks, 3.0 hrs per week 48

48

Topics Covered Extensively: Alternate Energy Sources Appropriate Technology; Biomass Conversion; Energy Conservation; Energy Conversion; Energy Storage; Heat and Energy Transfer; Intro. to Solar Energy; Passive Solar Technology; Photovoltaics; Domestic Hot Water; Swimming Pool Heating; Elec'l Generation, Central; Elec'l Generation, Small Scale; Process Heat, Agricultural; Process Heat, Industrial; Space Heating; Space Cooling; Wind Power, Central Systems; Wind Power, Small Systems

North Lake College [29066] Irving, Texas 75062

(214) $255-5229$

Programs and Curricula

Sular Energy Technician

Degree:

Contact:

AD, Solar Technology

Knowles, Jim

(214) 255-5325

Students Taking or Completing Offering: Solar Technician

Solar Related Courses

Energy Science I

Instructor:

Knowles, Jim

(214) $255-5260$

Department: Science/Math/Technology

Program or Curriculum: Solar Energy Technician

Credits

Student Level:

Duration:

Contact Hours

Freshman or Sophomore

16 Weeks, 6.0 hrs per week

Energy Transter

Future Technology

Instructor

Knowles, Jim

(211) 256-6260

Department: Science/Math/Technology

Program or Curriculum: Solar Energy Technician

Credits: 
Student Level:

Duration:

Contact Hours:

Freshman or Sophomore

16 Weeks, $3.0 \mathrm{hrs}$ per week

48

Classroom:

48

Topics Covered Extensively: Alternate Energy Sources; Appropriate Technology; Domestic Hot Water; Swimming Pool Heating; Elec'l Generation, Central; Elec'l Generation, Small Scale; Process Heat, Agricultural; Process Heat, Industrial; Spare r.nnling

Introduction to Solar

Instructor:

Knowles Jim

(214) $255-5260$

Department: Science/Math/Technology

Program or Curriculum: Solar Energy Technician

Credits:

2

Student Level:

Freshman or Sophomore

Duration:

Contact Hours:

16 Weeks; $2.0 \mathrm{hrs}$ per week

32

Classroom:

32

Topics Covered Extensively: Energy Conservation; Passive

Solar Technology

Materials - Material Handling

Instructor: Knowles, Jim

(214) $255-5260$

Department:

\section{Science/Math/Te}

Program or Curriculum: Solar Energy Technician

Credits: 3

Student Level: ' Freshman or Sophomore

Duration:

16 Weeks, $5.0 \mathrm{hrs}$ per week

Contact Hours:

80

Classroom:'

Laboratory:

64

Topics Covered Extensively: Plumbing Techniques; Sheet

Metal Techniques

Operational Diagnosis

Instructor:

Knowles, Jim

(214) 255-5260

Srience/Math/Technology

Department:

Solar Energy Technician

Crogram or Curriculum:

Student Level:

\section{3}

Freshman or Sophomore

niıration

Contact Hours:

16 Weeks. $4,0 \mathrm{hrs}$ per week

64

32

Laboratory:

32

Topics Covered Extensively: Solar Systems Design; Solar Systems Installation; Solar Systems Maintenance; Solar Syatems Testing and Evaluation

\section{Sizing Design and Retrofit}

Instructor:

Knowles, Jim

(214) 255-5260

Department:

Science/Math/Technology

Program or Curriculum: Solar Energy Technician

Credits:

Student Level: $\quad$ Freshman or Sophomore

Duration: $\quad 16$ Weeks, $7.0 \mathrm{hrs}$ per week

ronntart Hours: $\quad 112$

Classronm: $\quad 48$

Laboratory: $\quad 64$

Topics Covered Extensively: Solar Systems Design; Solar Systems installation

$\begin{array}{ll}\begin{array}{l}\text { Solar Codes and Consumerism } \\ \text { Instructor: }\end{array} & \text { Knowles, J. } \\ & (214) 255-5260 \\ \text { Department: } & \text { Science/Math/Technology } \\ \text { Program or Curriculum: Solar Energy Technician } & \\ \text { Credits: } & 2 \\ \text { Student Level: } & \text { Freshman or Sophomore } \\ \text { Duration: } & 16 \text { Weeks, 2.0 hrs per week } \\ \text { Contact Hours: } & 32 \\ \text { Classroom: } & 32\end{array}$

Topics Covered Extensively: Marketing/Market Analysis; Solar Energy Policy Development; Solar Economics; Solar Law/Legislation

Solar Practicum

Instructor:

Knuwles, Jin
(214) 255-5260

Department: Science/Math/Technology

Program or Curriculum: Solar Energy Technician

Credits: 5

Student Level: $\quad$ Freshman or Sophomore

Duration: 16 Weeks, 5.0 hrs per week

Contact Hours: $\quad 80$

Technical Survey of Energy Sources

Instructor: Knnwles, , I

(214) $255-5260$

Department: Science/Math/Technology

Program or Curriculum: Solar Energy Technician

Credits:

Student Level: $\quad$ Freshman or Sophomore

Duration: 16 Weeks

Topics Covered Extensively: Alternate Energy Sources;

Biomass Conversion; Energy Conservation; Energy Conversion; Wind Power, Central Systems

\section{Odessa College [3596] \\ Odessa, Texas 79760 \\ (915) $337-5381$ \\ Programs and Curricula \\ Solar Power \\ Contact: \\ Witcher, Norman \\ (915) $337-5381$}

Students Taking or Completing Offering: Solar Technician

\section{Solar Related Courses}

\section{Solar Power}

Instructor:

Witcher, Norman

Course Number:

(915) $337-5381$

Department:

R/AC 2300

Program or Curriculum: Solar Power

Credits:

Student Level:

Duration:

Contact Hours:

Refrigeration \& Air Conditioning

Classroom:

Laboratory:

6

Freshman or Sophomore

16 Weeks, 10.0 hrs per week. .

160

Topirs Coveren F.xtensively: Appropriate Technology:

Energy Storage; Heat and Energy Transfer; Intro. to Solar Energy: Solar systems Cumpuntuls, Sulai 3ystems

Design; Solar Systems Installation; Solar System Mainte-

nance; Space Heating

Number of Times Taught: 1

Dverage Fnrollment: 38

\section{Ranger Junior College [3603] \\ Ranger, Texas 76470 \\ (817) 647-3234}

Programs and Curricula

Air Conditioning \& Refrigeration - Solar Energy Option

Degree: AD, Applied Science

Contact: Stiles, Alton

(817) 647-32.34

Students Taking or Completing Offering: Solar Technician

\section{Solar Related Courses}

Air Cuinditioning and Refrigoration - VII

Course Number: $\quad$ AR 281

Department: Air Cond. \& Ref.

Program or Curriculum: $A / C$ and Refrig. - Sol. Ener. Option

Credits:

Student Level: Freshman or Sophomore .."

Topics Covered Extensively: Heat and Energy Transfer;

Solar System Components; Solar Collector Evaluation/

Design; Solar Systems Design; Solar Systems Installation:

Solar Systems Maintenance; Domestic Hot Water; Space

Heating; Space Conoling

\section{Fundamentals of Solar Heating \& Cooling}

Course Number: AR 263

Department: Air Cond. \& Retrig

Program or Curriculum: $A / C$ and Refrig. - Sol. Ener. Option 
Credits: 6

Student Level:

Freshman or Sophomore

Topics Covered Extensively: Energy Conversion; Energy

Storage; Heat and Energy Transfer; Intro. to Solar Energy;

Solar System Components; Solar Collector Evaluation/

Design; Solar Systems Design; Space Heating; Space

Cooling

Solar Thermal Energy Systems

Instructor:

Stiles, Alton

(817) $647-3234$

Course Number:

AR 264

Department:

Air Conditioning/Refrigeration

Program or Curriculum: $A / C$ and Refrig. - Sol. Ener. Option

Credits:

Student Level:

Duration:

Contact Hours:

Freshman or Sophomore

16 Weeks, $10.0 \mathrm{hrs}$ per week

160

32

room:

128

Laboratory:

vely: Plumbing Techniques: Solar

Systems Components; Solar Collector Evaluation/Design;

Solar Systems Design; Solar Systems Installation; Solar

Systems Maintenance; Solar Systems Testing and Evalua-

tion; Domestic Hot Water; Elec'l Generalion, Small Scale;

Space Heating; Space Cooling

Tyler Junior College [3648]

Tyler, Texas 75701

(214) $597-4281$

Programs and Curricula

Air Conditioning \& Refrigeration Technology

Degree: AD, Applied Science

Contact: Minter, Richard T.

(214) 593-1401

Students Taking or Completing Offering: Trade Specialty

Solar Related Courses

Introduction to Solar Systems

Instructor: Robinson, Carol T.

Course Number: $\quad$ (214) $592-8619$

Department: Technology

Program or Curriculum: Air Conditioning \& Refrig. Tech.

Credits: 3

Student Level: $\quad$ Freshman or Sophomore

Duration: $\quad 16$ Weeks, $3.0 \mathrm{hrs}$ per week

Contact Hours: $\quad 48$

Classroom: 48

Topics Covered Extensively: Intro, to Solar' Energy

Average Enrollment: $\quad 31$

\section{Solar Systems Installation}

Instructor:

Robinson, Carol T.

(?.14) 59 ? - 8619

$\begin{array}{ll}\text { Course Number: } & \text { AC 223S } \\ \text { Department: } & \text { Technology }\end{array}$

Program or Curriculum: Air Conditioning \& Refrig. Tech

Credits:

3

Student Level:

Duration:

Contact Hours:

Classroom:

Laboratory:

Topics Covered Extensively: Solar System Components:

Solar Systems Installation; Solar Systems Maintenance;

Solar Systems Testing and Evaluation; Domestic Hot

Water; Space Heating

Average Enrollment:

18

\section{UTAH}

Dixie College [3671]

Saint George, Utah 84770

(801) 6\%3-4811

Programs and Curricula
Solar Technology

Degree:

Contact:

Certificate of Completion

Hacking, John

(801) 673-4811

Solar Related Courses

Introduction to Applied Solar Energy

Instructor: Tait, Don

Course Number: ST 150

Department: Engineering Tech.

Program or Curriculum: Solar Technology

Credits:

Student Level: $\quad$ Freshman or Sophomore

Duration: $\quad 11$ Weeks, 5.0 hrs per week

Contact Hours: $\quad 55$

Classroom: $\quad 55$

Topics Covered Extensively: Energy Conversion; Energy

Storage; Heat and Energy Transfer; Intro. to Solar Energy;

Marketing/Market Analysis; Materials Research; Passive

Solar Technology; Plumbing Techniques; Solar System

Components; Solar Economics; Solar Home Construction;

Solar Collector Evaluation/Design; Solar Systems Design;

Solar Systems Installation; Solar Systems Maintenance:

Solar Systems Testing and Evaluation; Domestic Hot

Water; Space Heating; Space Cooling

Number of Times Taught: 12

Average Enrollment: 20

Solar Energy - Home Use Applications

Instructor: Tait, Don

(801) 673-4811

Course Number: ST 123

Department: Engineering Tech.

Program or Curriculum: Solar Technology

Credits: 3

Student Level: $\quad$ Freshman or Sophomore

Duration: $\quad 11$ Weeks, $3.0 \mathrm{hrs}$ per week

Contact Hours: $\quad 33$

Classroom: $\quad 16$

Laboratory: $\quad 15$

Topics Covered Extensively: Alternate Energy Sources;

Energy Conservation; Energy Conversion; Energy Storage; Intro. to Solar Energy; Passive Solar Technology; Plumbing Techniques; Solar System Components; Solar Economics; Solar Collector Evaluation/Design; Solar Systems Design; Solar Systems Installation; Solar

Systems Maintenance; Solar Systems Testing and Evaluation; Domestic Hot Water; Space Heating; Space Cooling

Number of Times Taught: 9

Average Ënrollment: $\quad 18$

\section{VERMONT, VIRGINIA}

None

\section{WASHINGTON}

North Seattle Community College [9704]

Seattle, Washington 98103

(206) 634-4444

Programs and Curricula

Heating - Solar Energy

Contact: Swenson, Don

(206) 634-4419

Students Taking or Completing Offering: Sheet Metal Worker

\section{Solar Related Courses}

\section{Heating - Solar Energy}

Instructor:

Swenson, Don

Department:

Engineering Technology

Student Level: High School Graduate

Duration: 6 Weeks, 15.0 hrs per week

Contart Hou,ss: $\quad 90$

Classroom: $\quad 45$

Laboratory: $\quad 45$ 
Topics Covered Extensively: Alternate Energy Sources: Appropriate Technology; Intro. to Solar Energy; Solar System Components; Solar Collector Evaluation/Design; Solar Systems Design; Domestic Hot Water; Space Heating

\section{Solar Energy}

Instructor:

Stepnich, Ivan

(206) 634-4423

Course iviumber.

ECT 207

Department:

Engineering Related Technologies

Program or Curriculum: Heating-Solar Energy

Credits:

3

Student Level:

Duration:

Freshman or Sophomore

11 Weeks, 3.0 hrs per week

Contact Hours:

33

Classroom:

3.3

Topics Covered Extensively: Alternate Energy Sources; Appropriate Technology; Energy Conservation; Energy Conversion; Energy Storage; Heat and Energy Transfer Intro. to Solar Energy; Passive Solar Technology; Plumbing Techniques; Solar System Components; Solar Home Construction; Solar Collector Evaluation/Design; Solar Systems Design; Solar Systems Installation; Solar

Systems Testing and Evaluation; Domestic Hot Water;

Elec'l Generation, Central; Process Heat, Industrial; Space

Heating

Number of Times Taught: 1

Average Enrollment: 25

\section{WEST VIRGINIA}

None

\section{WISCONSIN}

Moraine Park Technical Institute [9256] Fond Du Lac, Wisconsin 54935 (414) 922-8611

Programs and Curricula

Solar Energy

Contact:

Pasch, Rodney

(414) 922-8611

Students Taking or Completing Offering: Contractor, Do-ityourself Homeowner, Electrician, Plumber, Sheet Metal Worker

\section{Solar Related Courses}

Solar Applications for Construction Industry

rascin; R̈udriey

(414) $922-8611$

Course Number:

401-479

Department: Trade and Technical

Program or Curriculum: Solar Energy

Credits:

2

Student Level:

All levels

Duration:

1 Week, 6.0 hrs per week

Contact Hours:

6

Topics Covered Extensively: Intro. to Solar Energy; Solar Home Construction

Number of Times Taught: $s$

Average Enrollment: 80

\section{Solar Energy - Air Handling Systems}

Instructor: Pasch, R.

(414) $922-8611$

Course Number:

401-483

Department:

Trade and Technical

Program or Curriculum: Solar Energy

Credits:

\section{5}

All levels

Duration:

Contact Hours:

10 Weeks, 2.0 hrs per week

20

Classroom:

20

Topics Covered Extensively: Heat and Energy Transfer;

Solar System Components; Space Heating

Number of Times Taught: 2

Average Enroliment: $\quad 20$
Solar Energy for Realtors

Instructor: Pasch, $R$

(414) $922-8611$

Course Number: $\quad 401-425$

Department: Trade and Technical

Program or Curriculum: Solar Energy

Credits: $\quad 3$

Student Level: $\quad$ All levels

Duration: 2 Weeks, $5.0 \mathrm{hrs}$ per week

Contact Hours: $\quad 10$

Topics Covered Extensively: Alternate Energy Sources

Number of Times Taught: 1

Average Enrollment: $\quad 40$

Solar Energy Seminar

Pasch, $\mathrm{B}$

$\begin{array}{ll} & (414) 322-9611 \\ \text { Course Number: } & 401-482 \\ \text { Department: } & \text { Trade and Technical } \\ \text { Program or Curriculum: Solar Energy } \\ \text { Credits: } & 1 \\ \text { Student Level: } & \text { All levels } \\ \text { Duration: } & 1 \text { Week, } 4.0 \text { hrs per week } \\ \text { Contact Hours: } & 4 \\ \text { Classronm: } & 4\end{array}$

Topics Covered Extensively: Alternate Energy Sources Number of Times Taught: 2

Average Enrollment:

Solar Heat and Wind

Instructor:

100

Pasch, Rodney

Course Number: $\quad 401-480$

Department: Trade \& Technical

Program or Curriculum: Solar Energy

Credits:

5

Student Level:

Duration:

Contact Hours:

All levels

Topics Covered Extensively: Space Heating; Wind Power, Small Systems

Number of Times Taught: 5

Average Enrollment: 17

Wind Energy Applications

Pasch, R.

Course Number: $\quad$ (414) 922

Department: Trade arld Technical

Program or Curriculum: Solar Energy

Crendits: 2

Student Level: $\quad$ All levels

Duration: $\quad 1$ Week, $6.0 \mathrm{hrs}$ per week

Contact Hours: 6

Topics Covered Extensively: Elec'l Generation, Small

Scale; Wind Power, Small Systems

Number of Times Taught: 1

Average Enroliment: $\quad 40$

\section{WYOMING}

Sheridan College [3930]

Sheridan, Wyoming 82801

(307) 674-6446

Program and Curricula

Solar Energy Technology

Degree:

AD, Engineeririg Technology -

Contact:

Solar Option

Ohm, Kenneth $R$

(307) 674-6446

Students Taking or Completing Offering: Installer-

Residential (Solar System), Installer-Commercial (Solar

System), Solar Technician, Do-it-yourself Homeowner

Solar Related Courses

Energy Storage

Course Number: 152

Program or Curriculum: Solar Energy Technology

Student Level: 
Topics Covered Extensively: Energy Storage; Photo-

voltaics; Wind Power, Small Systems

Installation and Service - Solar System

Course Number: $\quad 158$

Program or Curriculum: Solar Energy Technology

Student Level: $\quad$ Freshman or Sophomore

Topics Covered Extensively: Solar System Components;

Solar Collector Evaluation/Design; Solar Systems Installa-

tion; Solar Systems Maintenance; Solar Systems Testing

and Evaluation; Space Heating; Space Cooling

Intro. to Solar Heating \& Cooling

Course Number: $\quad 150$

Program or Curriculum: Solar Energy Technology

Student Level: $\quad$ Freshman or Sophomore

Topics Covered Extensively: Intro. to Solar Energy; Materials Research; Solar System Components; Solar Collector Evaluation/Design; Solar Systems Design; Domestic Hot Water; Elec'l Generation, Small Scale; Space Heating;

Space Cooling

Solar Collectors

Course Number:

151

Program or Curriculum: Solar Energy Technology

Student Level: $\quad$ Freshman or Sophomore

Topics Covered Extensively: Solar Systerii Cumponents;

Solar Collector Evaluation/Design; Solar Systems Design;

Solar Systems Testing and Evaluation

Solar Energy Fundamentals

Instructor:

Ohm, Kenneth R.

(307) 674-6446

Course Number: $\quad 19-190$

Department: Career/Tech

Program or Curriculum: Solar Energy Technology

Credits: 3

Student Level: $\quad$ Freshman or Sophomore

Duration: $\quad 16$ Weeks, $4.0 \mathrm{hrs}$ per week

Contact Hours: $\quad 60$

Classroom: $\quad 48$

Laboratory:

48

Topics Covered Extensively: Alternate Energy Sources;

Appropriate Technology; Energy Storage; Heat and

Energy Transfer; Intro. to Solar Energy; Solar Home Construction; Solar Collector Evaluation/Design; Solar

Systems Design; Solar Systems Installation; Solar

Systems Maintenance; Domestic Hot Water; Process Heat, Industrial; Space Heating: Space Cooling; Wind Power,

Central Systems; Wind Power, Small Systems

Number of Times Taught: 2

Average Enrollment: $\quad 35$

Solar Heating and Cooling Systems

Course Number: 155

Program or Curriculum: Solar Energy Technology

Student Level: Freshman or Sophomore

Topics Covered Extensively: Energy Storage; Solar System

rnmponents; Solar Collector Evaluation/Designr, 3ulaı

Systems Design; Domestic Hot Water; Process Heat, Agricultural; Process Heat, Industrial; Space Heating; Space

Cooling

Wind Systems

Course Number:

153

Prograiri or Curriculum: Solar Energy Technology

Student Level: Freshman or Sophomore

Topics Covered Extensively: Wind Power, Central Systems; Wind Power, Small Systcms 
Adirondack Community College.......... 2

Antioch University/West......................

Barton County Community Junior College.

Beaufort Technical Education Center.

Bismarck Junior College

Brevard Community College.

Cabrillo College.

California State University - Sonoma.. Cambridge School -

Weston Center of Open Education

Cape Cod Community College.

Cape Fear Technical Institute

Cayuga County Commuinity coilege.

Center for Employment Training.........

Central Texas Collegc.

Cerro Coso Community College..........

Chaffey College.

Charles S. Molt Community College...

Clark County Community College......

Coastline Community College.

Colorado Technical College..

Cornerstones, Wing School of Shelter Technology

Cosumnes River College...

CUNY New York City Community College.

Dekalb Community College.

Denver, Red Rocks Campus Community Collcge of.

Des Moines Area Community College.

Dixie College.

Fscex rnunty Technica!

Careers Center....

Evergreen Valley College.

Ferris State College.

Florence Darlington

Technical College.

Fiorida Solar Energy Center.

Grand Rapids Junior College.

rulf Coast Community College

Illinois Eastern Community College OIney Central Campus.

Kansas Technical Institute.

Keystone Junior College.

Lansing Community College.

Linn-Benton Community College......

Long Beach City College.

Mercer County Area

Vocational Technical Schools......

Metropolitan Technical

Community College.

Miami-Dade Community College.......

Mississippi County

Community College.

Modesto Junior College

Mohawk Valley Community College.. \begin{tabular}{l|l|l} 
Monterey Peninsula College................ & 6
\end{tabular}

Moraine Park Technical Institute......... $\quad 34$

Motlow State Community College....... 29

Mount San Antonio College.................. 6

Muscatine Community College........... 14

National Training Fund....................... 11

Navarro College..

New 'England Fuel Institute.................. 17

New'Hampshire Vocational

Technical College Manchester.......... 20

Newcastle County Vocational

Technical School............................ 11

NHAW - Home Study Institute.............. 26

North Dakota Siate

School of Sclence..............................

North Lake College............................. 31

North Seattle Community College....... $\quad 33$

Noitheast Institute of lindustrial Technology......................

Ocean County Vocational

Technical Schools.

Odessa College

Office of Appropriate Technology........

Otéro Junior College..

Peňnsylvania Institute of Technology..

Peñnsylvania Stale University

Shenango Valley Campus................. $\quad 27$

Peńsacola Junlor College..................... 12

Ranger Junior Collcge......................... $\quad 32$

Reidwoods, Colloge of the....... . .. 6

RETS Tech Center................................. 16

San Diego Community CollegeCity College.

San Dltyu Cummininity Collogo

Evening College.

San Jose City College.

Scolt Community College...

Shelter Institute...

Sheridan College.

Solar Store Incorporated..

Solar Technician Training Program-

Office of Appropriáte li echnoiogy.....

Solarcon

Soulh Florida Technical Institute.........

Southern New Jersey OIC

Springfield Technical

Community College.

SUNY Agriculture and Technical College at Delhi.

Triangle Institute

of Technology, Incorporated............ $\quad 28$

Trident Technical College..................... 28

Tyler Junior College..

Western lowa Technical....................... 14

Yavapai College................................... 1

York Technical College...................... $\quad 28$ 
Distributed by:

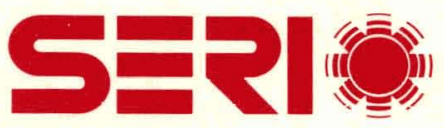

\section{Solar Energy Research Institute}

1536 Cole Boulevard

Golden, Colorado 80401

Operated for the U.S. Department of Energy

by Midwest Resea:ch Institute 